Marquette University

e-Publications@Marquette

$1-1-1997$

\title{
Charge-Transfer Probes for Molecular Recognition via Steric Hindrance in Donor-Acceptor Pairs
}

Rajendra Rathore

Marquette University

Sergey V. Lindeman

Marquette University, sergey.lindeman@marquette.edu

Jay K. Kochi

University of Houston

Accepted version. Journal of the American Chemical Society, Vol. 119, No. 40 (1997): 9393-9404.

DOI. (C) 1997 American Chemical Society. Used with permission.

Sergey V. Lindeman and Rajendra Rathore were affiliated with the University of Houston at the time of publication. 
Marquette University

e-Publications@Marquette

\title{
Chemistry Faculty Research and Publications/College of Arts and Sciences
}

This paper is NOT THE PUBLISHED VERSION; but the author's final, peer-reviewed manuscript. The published version may be accessed by following the link in the citation below.

Journal of the American Chemical Society, Vol. 119, No. 40 (October 8, 1997): 9393-9404. DOI. This article is (C) American Chemical Society and permission has been granted for this version to appear in ePublications@Marquette. American Chemical Society does not grant permission for this article to be further copied/distributed or hosted elsewhere without the express permission from American Chemical Society.

\section{Charge-Transfer Probes for Molecular Recognition via Steric Hindrance in Donor-Acceptor Pairs}

\author{
R. Rathore
}

Department of Chemistry, Marquette University, Milwaukee WI

S. V. Lindeman

Department of Chemistry, Marquette University, Milwaukee WI

J. K. Kochi

Department of Chemistry, University of Houston, Houston, TX

\section{Abstract}

Molecular association of various aromatic hydrocarbons ( $D$, including sterically hindered donors) with a representative group of diverse acceptors ( $A=$ quinone, trinitrobenzene, tetracyanoethylene, tropylium, tetranitromethane, and nitrosonium) is visually apparent in solution by the spontaneous appearance of distinctive colors. Spectral (UV-vis) analyses of the colored solutions reveal their charge-transfer origin $\left(\lambda_{\mathrm{C} T}\right)$, and they provide quantitative 
information of the intermolecular association in the form of the $K_{\mathrm{DA}}$ and $\varepsilon \mathrm{CT}$ values for the formation and visualization, respectively, of different $[D, A]$ complexes. Importantly, such measurements establish charge-transfer absorption to be a sensitive analytical tool for evaluating the steric inhibition of donor-acceptor association. For example, the steric differences among various hindered aromatic donors in their association with quinone are readily dramatized in their distinctive charge-transfer (color) absorptions and verified by X-ray crystallography of the charge-transfer crystals and/or QUANTA molecular modeling calculations of optimum intermolecular separations allowed by van der Waals contacts.

\section{Introduction}

Molecular recognition and preassociation are conceptually vital to catalytic stereospecificity and other contemporaneous topics in organic chemistry like self-assembly and organization, supramolecular (host-guest) chemistry, etc. $\stackrel{1-3}{-3}$ Of the various measures available for the quantitative evaluation of intermolecular interactions in solution - especially weak nonbonded ones - the appearance of new spectral bands arising from the charge-transfer (CT) transitions between electron donors and electron acceptors is especially useful and easy to apply. ${ }^{4-6}$ Indeed, the ubiquitous CT absorptions are diagnostic of a very wide spectrum of intermolecular electron donor-acceptor (DA) interactions arising in extremely stable, isolable 1:1 complexes on one hand,,$\underline{7}$ to highly transient complexes (with collisional lifetimes) at the other extremum. $\underline{8}$ From a structural point of view, however, it is not at all clear a priori what the critical donor-acceptor encounter (distance) must be for the relevant charge-transfer absorptions to be in evidence. $\underline{9}$

In order to establish the limits to which charge-transfer is applicable as an analytical probe for intermolecular interactions, we employ in this study four classes of aromatic donors (Chart 1), in which the essential benzenoid (donor) $\pi$-chromophore is sterically encumbered to various degrees by increasing alkyl bulk. Thus, the first (simply methylated) member in each class represents the sterically most accessible donor, and all members in each class are of comparable donor strengths. $\underline{\underline{10}}$

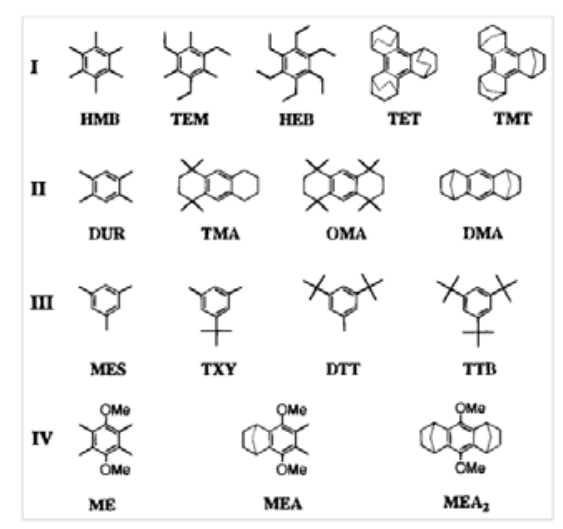

Chart 1 
In a complementary way, we identify two classes of sterically graded electron acceptors in Chart 2. As such, the planar $\pi$-acceptors are presented in the order of their increasing size from tetracyanoethylene to 1,3,5-trinitrobenzene. Among the $\sigma$-acceptors, the diatomic nitrosonium cation and dibromine are the smallest and least subject to steric hindrance, especially in comparison with the larger, tetrahedral acceptors tetranitromethane and carbon tetrabromide. The relative acceptor strengths in Chart 2 are indicated by the trend in the reduction potentials $\left(\mathrm{E}^{\circ}\right.$ red $\mathrm{V}$ vs SCE). $\underline{11,12}$

Donor-acceptor pairs in solution are quantitatively monitored in this study by UV-vis spectral changes, and the relevant charge-transfer interactions identified in X-ray crystal structures and compared with molecular modeling calculations. Steric effects are not only exploited in the structural requirements for the intermolecular formation of 1:1 donor-acceptor complexes but also to achieve intramolecular selectivity in biaryls and in a tethered donor containing more than one aromatic center.

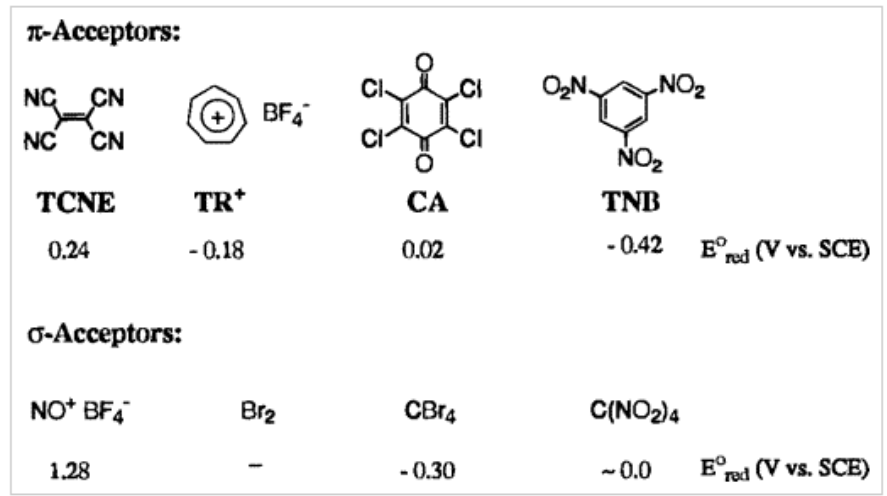

\section{Chart 2}

\section{Results and Discussion}

I. Visual Detection of Intermolecular Donor-Acceptor Associations. Exposure of the various methylbenzenes in Chart 1 to the prototypical m-acceptor chloranil (CA) spontaneously resulted in brightly colored dichloromethane solutions. The colorations progressively evolved from yellow (mesitylene) to orange (durene) to purple (hexamethylbenzene) in line with the decreasing ionization potentials of the aromatic donors. ${ }^{10}$ Similar vivid colorations were also observed when the same methylbenzenes were mixed with other electron acceptors such as 1,3,5-trinitrobenzene (TNB), and further red-shifts in colors occurred with the stronger $\pi$ acceptor tetracyanoethylene (TCNE) as well as the tropylium cation $\left(\mathbf{T R}^{+}\right)$in Chart 2.

II. Quantification of Donor-Acceptor $(\pi-\pi)$ Associations. The UV-vis spectral changes in Figure 1 typically show the monotonic growth of the diagnostic charge-transfer absorbance with its visible maximum at $\lambda_{\mathrm{CT}}=520 \mathrm{~nm}$ upon the incremental addition of hexamethylbenzene (HMB in Chart 1) to a solution of chloranil (CA) in dichloromethane at $24^{\circ} \mathrm{C}$. This well-resolved (featureless) absorption band was ascribed to the intermolecular donor-acceptor association, i.e. 


$$
\mathrm{HMB}+\mathrm{CA} \stackrel{K_{\text {ga }}}{\rightleftharpoons}[\mathrm{HMB}, \mathrm{CA}]
$$

in which the characteristic color derives from the charge-transfer transition, as originally formulated by Mulliken. ${ }^{4}$ Such a structural assignment was readily verified by the isolation of dark purple crystals of the 1:1 complex in $>95 \%$ yield from an equimolar solution of hexamethylbenzene and chloranil, simply by the very slow removal of dichloromethane in vacuo. X-ray crystallography of the charge-transfer crystal revealed the hexamethylbenzene to be directly juxtaposed on the chloranil and separated by an interplanar distance of $d=3.51 \AA$, as illustrated in the top and side perspectives $A_{T}$ and $A_{s}$ (all hydrogens omitted for clarity). ${ }^{13,14}$ Indeed, the observed donor-acceptor separation in the purple crystal is remarkably close to the calculated distance of $d=3.57 \AA$ by energy minimization of the intermolecular van der Waals contacts between hexamethylbenzene and chloranil. The predicted structure based on the QUANTA molecular modeling analysis ${ }^{15}$ is shown in the space-filling representation $\mathbf{B}$ below.
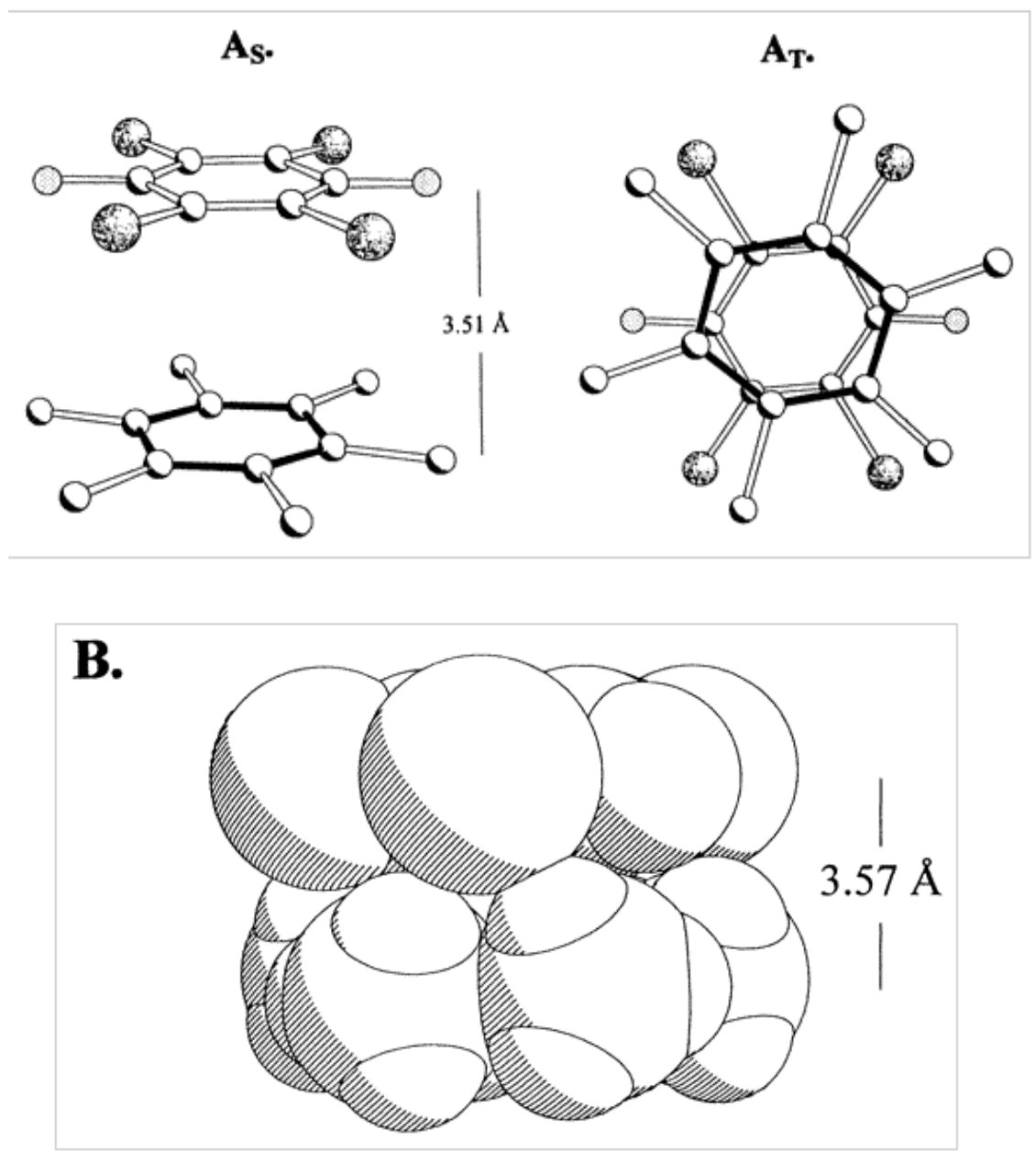

For the quantitative analysis of the donor-acceptor association in solution, the spectrophotometric absorbance changes in Figure 1 were treated by the Benesi-Hildebrand procedure, $\underline{\underline{16}}$ i.e. 


$$
\frac{[\mathrm{CA}]}{A_{\mathrm{CT}}}=\frac{1}{\epsilon_{\mathrm{CT}}}+\frac{1}{K_{\mathrm{DA}} \epsilon_{\mathrm{CT}}} \frac{1}{[\mathbf{D}]}
$$

where $A_{c t}$ is the molar absorbance and $\varepsilon_{c t}$ is the extinction coefficient of the charge-transfer band at the monitoring wavelength (generally close to $\lambda_{\max }$ ). For hexamethylbenzene (D) concentrations much greater than that of chloranil, a plot of [CA]/Act vs the reciprocal donor concentration was linear, and the least-squares fit produced a correlation coefficient of greater than 0.999 in the inset of Figure 1. From the slope $\left[K_{\mathrm{DA \varepsilon CT}}\right]^{-1}$ and the intercept $[\varepsilon \mathrm{cT}]^{-1}$, the values of the association constant and the extinction coefficient were readily extracted as $K_{\mathrm{DA}}$ $=2.8 \mathrm{M}^{-1}$ and $\varepsilon_{520}=2800 \mathrm{M}^{-1} \mathrm{~cm}^{-1}$, respectively. Such a limited magnitude of $K_{\mathrm{DA}}$ for hexamethylbenzene and chloranil indicated that the donor-acceptor interaction is described as weak at best $\left(\Delta G^{\circ} D A=-0.6 \mathrm{kcal} \mathrm{mol}^{-1}\right)$, as typical for the spontaneous formation of electron donor-acceptor complexes of quinones (and other m-electron acceptors) with various types of other electron donors (such as alkenes, enol ethers, sulfides, etc.). $\underline{.17}$

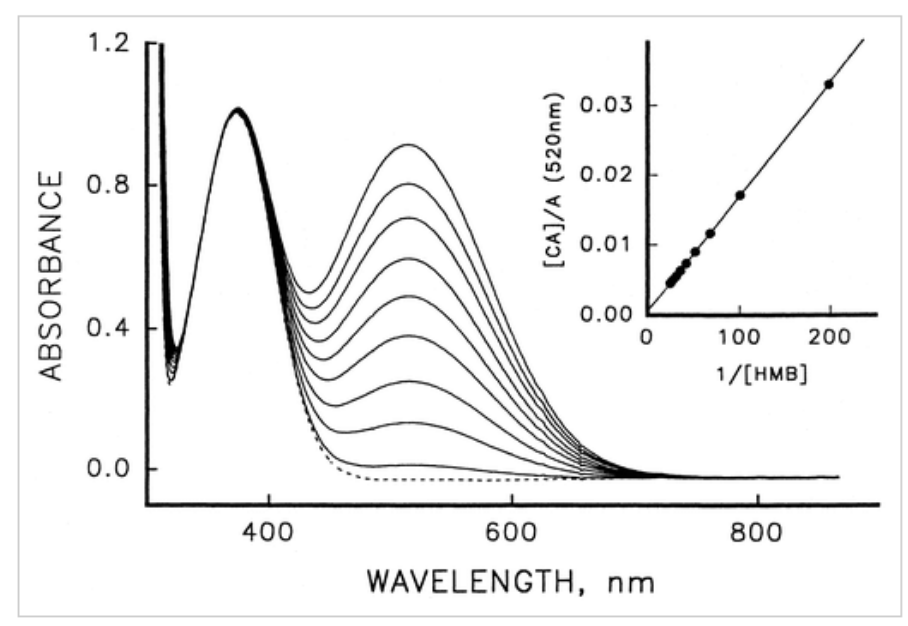

Figure 1 Spectral (UV-vis) changes attendant upon the incremental addition of hexamethylbenzene to chloranil in dichloromethane. Inset: Benesi-Hildebrand plot.

The donor-acceptor interactions of the homologous methylbenzenes in Chart 1 with chloranil showed a progressive red-shift of the charge-transfer band $\lambda_{C T}$ for HMB $>$ DUR $>$ MES, as listed in Table 1 (column 4). The same trend was observed in the association constants $K_{\mathrm{DA}}$, but the magnitude of the change was somewhat limited (see column 5). Weak but distinctive donor-acceptor interactions of hexamethylbenzene were also indicated by the comparison of the charge-transfer absorptions $\left(\lambda_{\mathrm{C} T}\right)$ and the magnitudes of the formation constants $\left(K_{\mathrm{DA}}\right)$ with the other $\pi$-acceptors including trinitrobenzene, tropylium, and tetracyanoethylene, as listed in Tables 1 and 4. For comparative purposes, the intensities of the CT (color) absorptions of the various donor-acceptor complexes in Table 1 are given by the values of $K_{\mathrm{DA}} \varepsilon_{\mathrm{C} T}$ in column 7. 18 
Table 1. Donor-Acceptor Association of Various Methylbenzenes with Different $\pi$-Acceptors ${ }^{a}$

DONOR

$\begin{array}{llllll}\begin{array}{lll}\text { [IP, eV] } \\ \left(\mathrm{E}_{\mathrm{ox}}, \mathrm{V}\right)\end{array} & \text { Acceptor } & \begin{array}{c}\lambda_{\mathrm{CT}} \\ (\mathrm{nm})\end{array} & \begin{array}{c}K_{\mathrm{DA}} \\ \left(\mathrm{M}^{-1}\right)\end{array} & \begin{array}{c}\varepsilon_{\mathrm{CT}} \\ \left(\mathrm{M}^{-1} \mathrm{~cm}^{-1}\right)\end{array} & K_{\mathrm{DA}} \varepsilon_{\mathrm{CT}} \\ & & & \end{array}$

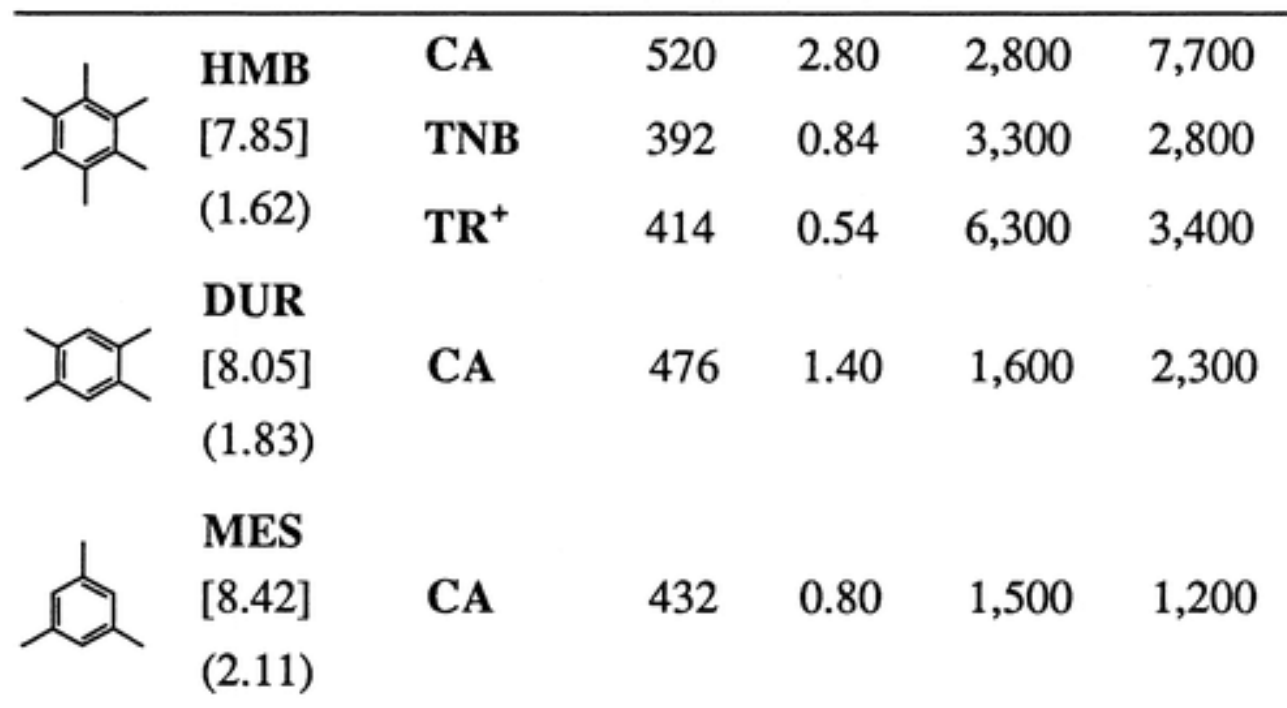

${ }^{a}$ In dichloromethane containing 4-6 mM acceptor and 50-500 mM aromatic donors at $24{ }^{\circ} \mathrm{C}$.

III. Complete Steric Inhibition of Donor-Acceptor ( $\pi-\pi)$ Association. Analogous exposure of hexaethylbenzene (HEB) to chloranil led to no coloration, and no new CT absorption band was observed in the colorless dichloromethane solution even in the presence of a large excess of HEB. Furthermore, many attempts to isolate charge-transfer crystals of the HEB complex with chloranil in various molar ratios from dichloromethane, chloroform, acetone, ethyl acetate, etc. were all unsuccessful, and low-temperature crystallization merely led to phase separation of the individual (pure) components. Such a striking difference between hexaethylbenzene and hexamethylbenzene (vide supra) was not restricted to chloranil. Thus, all other m-acceptors including trinitrobenzene, tropylium, and tetracyanoethylene showed distinctive CT colorations ranging from yellow to orange to green when mixed with hexamethylbenzene, whereas no (or very faint) colorations were detected with hexaethylbenzene at even higher concentrations. The UV-vis spectral changes in Figure 2 confirmed that neither chloranil nor trinitrobenzene participated in charge-transfer association with hexaethylbenzene, and the smallest acceptors tetracyanoethylene and tropylium showed (at best) very weak CT interactions with HEB (Table 2). $.19,20$

The comparative charge-transfer behaviors notwithstanding, the intrinsic electron-donor properties of HEB are even somewhat better than those of HMB insofar as their relative oxidation potentials of $E_{\mathrm{ox}}{ }^{\circ}=1.59$ and $1.62 \mathrm{~V}$ vs SCE, respectively. ${ }^{10}$ In order to clarify this anomalous variation (of $\lambda_{C T} V S E_{0 x^{\circ}}$ ) between HMB and HEB, we synthesized the novel hybrid triethylmesitylene (TEM) with an intermediate oxidation potential of $E_{0 x^{\circ}}=1.61 \mathrm{~V} v s$ SCE. In 
fact, the exposure of TEM to chloranil immediately led to the characteristic purple coloration of the charge-transfer association $\left(\lambda_{\mathrm{CT}}=516 \mathrm{~nm}\right)$, and dark purple crystals of the EDA complex were readily isolated in high yields from an equimolar mixture of triethylmesitylene and chloranil. Indeed, the charge-transfer parameters of the purple [TEM, CA] were essentially identical to those of the HMB analogue. Further comparisons of the TEM and HMB complexes with trinitrobenzene, tropylium, and tetracyanoethylene in Tables 1 and 2 established the same similarities. However, the association constants of the TEM complexes (Table 2, column 5) were always roughly half the value of $K_{\mathrm{DA}}$ for the corresponding HMB complex (Table 1 ) in dichloromethane. The latter was consistent with a statistical factor of 0.5 for only half the faces available for association, as established by the unique conformation of TEM in the chloranil complex shown below in structure $\mathbf{C}$ by $\mathbf{X}$-ray crystallography. Such a tripodal arrangement of all three ethyl groups on the opposite face of TEM is directly related to the conformation of the ethyl groups on both faces of HEB with quasi $D_{3 d}$ symmetry in structure $\mathbf{D}$ which was previously established by Mislow and co-workers, ${ }^{21}$ i.e.
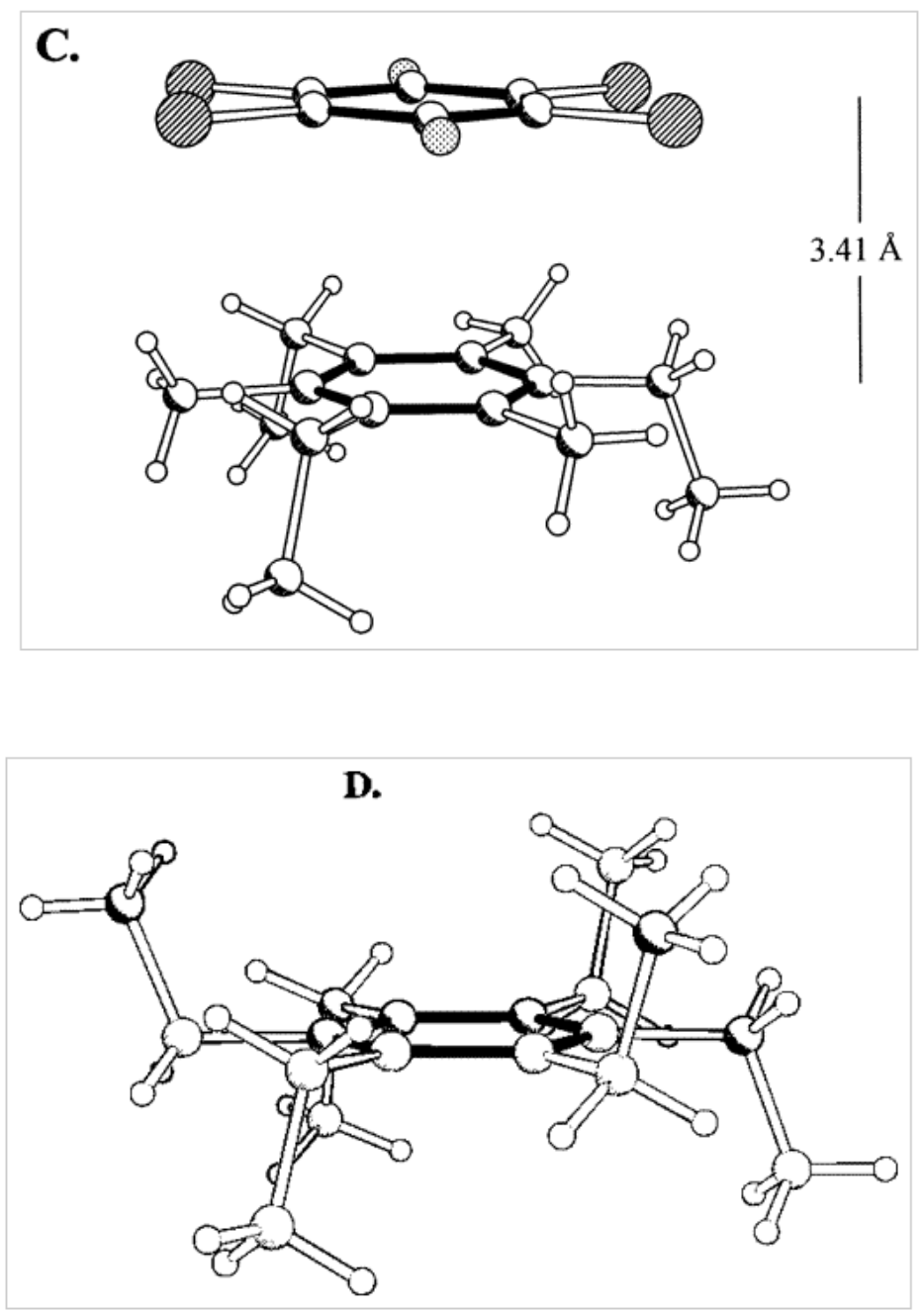
Table 2. Donor-Acceptor Association of Sterically Hindered Hexaalkylbenzenes with $\pi-$ Acceptors $^{a}$

\section{DONOR}

[IP, eV]

$\left(E_{\mathrm{ox}}, \mathrm{V}\right)$

Acceptor

$\lambda_{\mathrm{CT}}$

(nm)

$K_{\mathrm{DA}} \quad \varepsilon_{\mathrm{CT}} \quad K_{\mathrm{DA}} \varepsilon_{\mathrm{CT}}$

$\left(\mathrm{M}^{-1}\right) \quad\left(\mathrm{M}^{-1} \mathrm{~cm}^{-1}\right)$

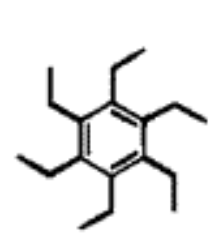

HEB

CA $\quad$ c - $<0.008^{\mathrm{d}}$

$1,000^{\mathrm{d}}<10^{\mathrm{d}}$

[7.71]

TNB

370

$\sim 0.02$

$\sim 3,000 \sim 50$

(1.59) $\mathbf{T R}^{+}$

$376 \sim 0.02$

$\sim 2,000 \sim 40$

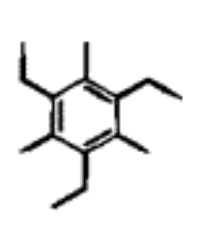

TEM

CA

516

0.90

$1,800 \quad 1,600$

$[7.88]^{\mathrm{d}}$

TNB

389

0.31

$3,500 \quad 1,000$

(1.61) $\mathbf{T R}^{+}$

408

0.41

$6,000 \quad 2,500$

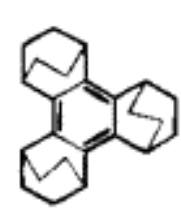

CA $\quad$ c - $\quad<0.008^{\mathrm{d}}$

$1,000^{\mathrm{d}}<10^{\mathrm{d}}$

\section{[-b-]}

TNB

-c -

$<0.004^{\mathrm{d}}$

$3,000^{d}<10^{d}$

(1.53)

TR $^{+}$

-c - $<0.005^{\mathrm{d}}$

$2,000^{\mathrm{d}}<10^{\mathrm{d}}$

a See Table 1. ${ }^{b}$ Not determined. ${ }^{c}$ No new absorption band. ${ }^{d}$ Estimated values, see Experimental Section.

From such a comparative behavior of HMB, TEM, and HEB with chloranil as well as the other $\pi$-acceptors TNB, TR ${ }^{+}$, and TCNE it is easy to conclude that a group of three $(1,3,5)$ ethyl substituents is sufficient for the complete steric inhibition of the face of a benzenoid (donor) chromophore for intermolecular association by a $\pi$-acceptor. Moreover, the comparable steric inhibition is achieved by tris-annulations at the $\alpha$-carbons, as presented in the bicyclic structure TET (see Table 2, last 3 entries) and the homologous TMT. Energy minimization between chloranil and HEB was predicted with the aid of QUANTA molecular modeling calculations to occur at an interplanar distance of $d=4.50 \AA$ in structure $\mathbf{E}$, and the predicted separation of chloranil and TET was $d=4.51 \AA$ in structure F. As such, the difference of $0.9 \AA$ between the observed separation of $3.6 \AA$ in [HMB, CA] and the predicted separation of $4.5 \AA$ in $[\mathrm{HEB}, \mathrm{CA}]$ could represent a "gray" area in which very weak, but visually (color) and spectrally (CT) observable association may be apparent. In order to pursue this possibility, we synthesized a series of unsymmetrical aromatic donors designed to cover the benzenoid face only partially. 

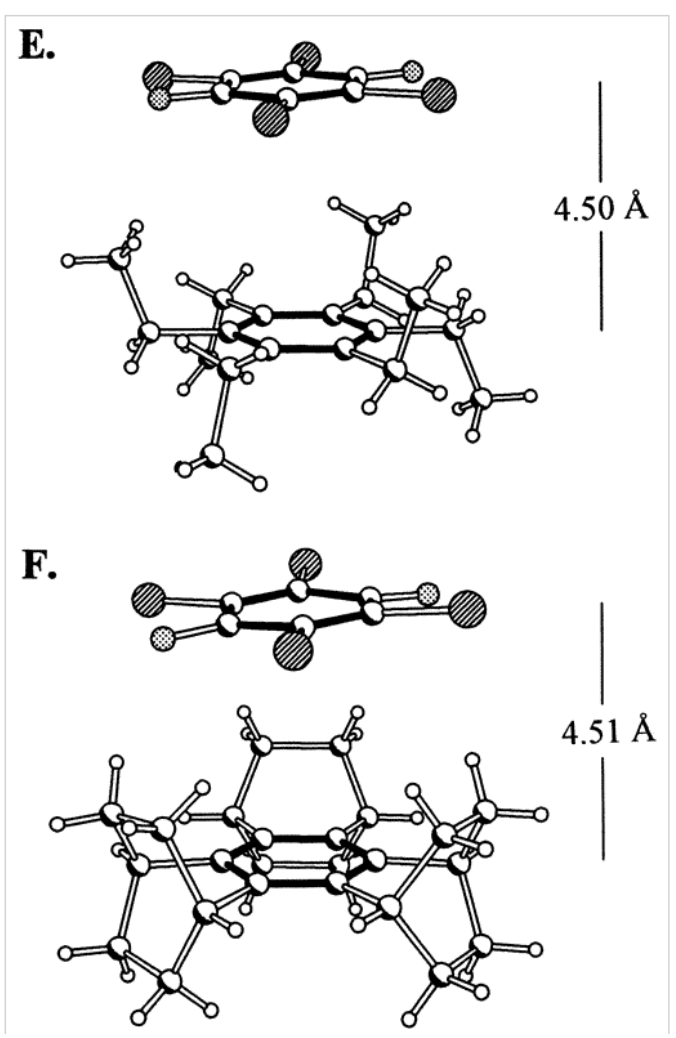

IV. Steric Modulation of Donor-Acceptor ( $\pi-\pi)$ Associations. Mesitylene (MES) yielded a bright yellow solution when exposed to chloranil, but 1,3,5-tri-tert-butylbenzene (TTB) under the same conditions, as expected, led to no coloration. However, intermediate behavior was shown by the homologous tert-butylxylene (TXY) which afforded a very pale yellow solution with chloranil, and the coloration with di-tert-butyltoluene (DTT) was barely discernible. The

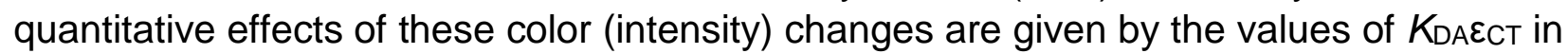
Table 3 (column 6), ${ }^{20}$ which were obtained from the spectrophotometric analysis of the chargetransfer absorptions (Figure 3A) attendant upon the incremental additions of these aromatic donors to chloranil in dichloromethane. The qualitative trend of the color intensity followed the monotonic decrease in the association constant KDA with increasing number of tert-butyl groups. $\underline{22}$ However, the latter had no significant effect on the intrinsic donor strength, since the values of the oxidation potentials $E_{0 x}{ }^{\circ}$ tabulated in column 2 were uniformly invariant. This observation, taken together with the constancy of the charge-transfer transition $\left(\lambda_{\mathrm{ct}}\right.$ in column 3), indicated that the interplanar separations (d) between the chloranil and MES, TXY, and DTT in the donor-acceptor associations were all comparable ${ }^{23}$ despite the decreasing strength of the interaction $\left(\triangle G_{D A}\right)$. In order to identify the origin of the difference, we carried out the QUANTA molecular modeling analysis of the nonbonding interactions in all four donor-acceptor associations. It is particularly noteworthy that energy minimization was predicted to occur at essentially the same interplanar separation of $d=3.4 \AA$ in the chloranil complex with MES, TBX, and DTT in structures $\mathbf{G}, \mathbf{H}$, and $\mathbf{J}$, respectively, but at a significantly larger separation of $d=4.5 \AA$ for tri-tert-butylbenzene in structure K. Such a donor-acceptor association in the mono- and di-tert-butyl-substituted donors was achieved by a small parallel shift of chloranil away from the tert-butyl group(s). ${ }^{24}$ 

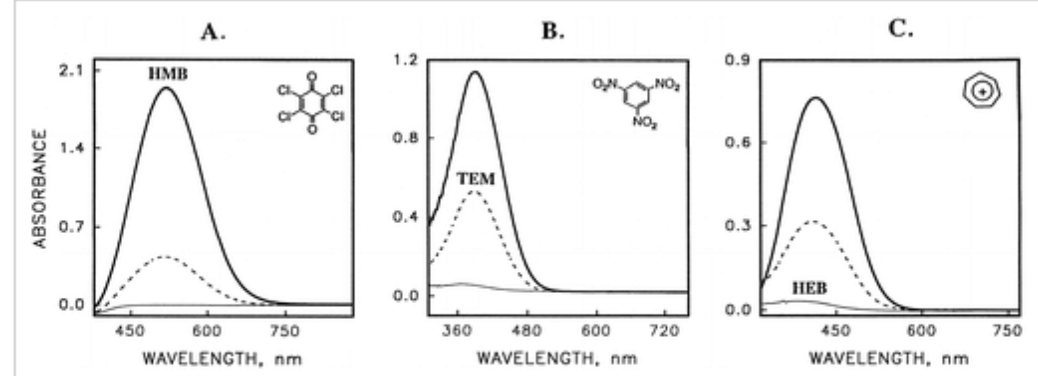

El view hi-res image $\overline{\text { ppt }}$ download to MS PowerPoint

Figure 2 Steric hindrance in the CT absorption bands of HMB (thick line), TEM (dashed line), and HEB (thin line) complexed with (A) chloranil, (B) trinitrobenzene, and (C) tropylium obtained by spectral subtraction of the uncomplexed acceptor from the experimental UV-vis spectra for (A) $6 \mathrm{mM}$ CA with $50 \mathrm{mM} \mathrm{HMB}, 50 \mathrm{mM}$ TEM , and $500 \mathrm{mM} \mathrm{HEB}$ in $\mathrm{CH}_{2} \mathrm{Cl}_{2}$, (B) 5 mM TNB with $100 \mathrm{mM} \mathrm{HMB}, 100 \mathrm{mM}$ TEM, and $500 \mathrm{mM}$ HEB in $\mathrm{CH}_{2} \mathrm{Cl}_{2}$, and (C) 9 or (4.5) $\mathrm{mM} \mathrm{TR}^{+} \mathrm{BF}_{4}^{-}$with $22 \mathrm{mM} \mathrm{HMB}, 22 \mathrm{mM}$ TEM, and $(22 \mathrm{mM})$ HEB in $\mathrm{CH}_{3} \mathrm{CN}$.
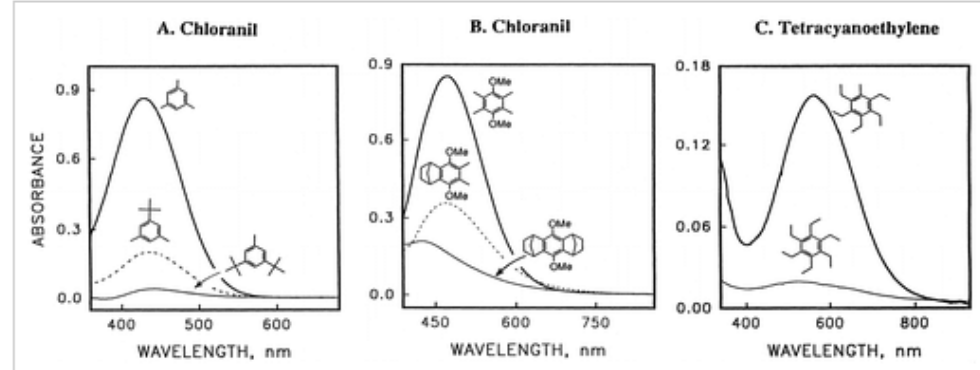

Figure 3 Partial steric hindrance in donor-acceptor association of (A) $4 \mathrm{mM} \mathrm{CA}$ with $250 \mathrm{mM}$ MES, TXY, and DTT, (B) 4 mM CA with $240 \mathrm{mM}$ ME, MEA, and MEA2, and (C) $1.7 \mathrm{mM}$ TCNE with $102 \mathrm{mM}$ PET and HEB in dichloromethane, obtained as difference spectra analogous to those in Figure 2.

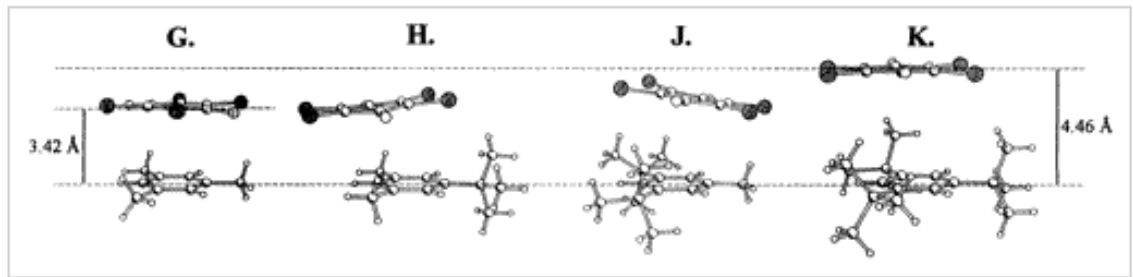

Indeed, such limited lateral displacements along the aromatic planes are not expected to be important factors in the charge-transfer transitions to significantly alter the values of $\lambda_{\mathrm{cr}}{ }^{25}$ 
Table 3. Donor-Acceptor Association of the Class II, III, and IV Aromatic Donors with Chloranila

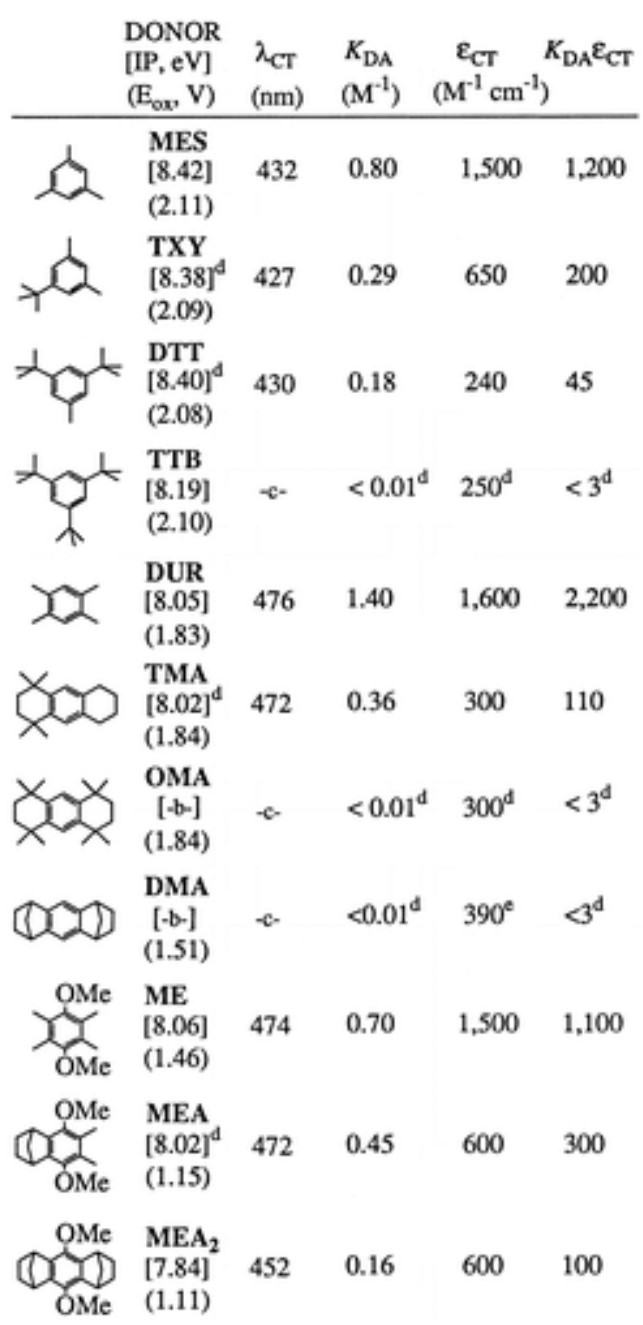

${ }^{a}$ From $4 \mathrm{mM} \mathrm{CA}$ and $0.01-1 \mathrm{M}$ donor in dichloromethane at $24^{\circ} \mathrm{C} \cdot{ }^{b-d}$ See Table 2.

Among the class II donors (based on durene in Chart 1), the tetramethyl derivative TMA showed the same "partial" steric behavior relative to the completely hindered octamethyl analogue OMA (and the bicyclic version DMA) at the other extremum. For example, the results in Table 3 (entry 6) point to the strongly diminished donor-acceptor interaction of TMA relative to DUR in its association with chloranil, ${ }^{26}$ in a manner similar to the differentiation of TXY and MES in the tert-butyl series (class III). Similarly, the bis-annulated donors OMA and DMA were subject to complete steric inhibition, much like tri-tert-butylbenzene in class III and TET and TMT in class $\mathrm{I}$.

The replacement of a pair of methyl groups in HMB with methoxy groups render the class IV aromatic ethers to be the best $\pi$-donors by virtue of the low $E_{0 x}{ }^{\circ}$ values in Table $3 .{ }^{27}$ As such, these methyl ethers were more tolerant to steric encumbrance - the bis-annulated MEA $\mathbf{A}_{2}$ being 
much less subject to partial steric hindrance than its counterpart DMA in class II, as shown by a direct comparison of $K_{\mathrm{DA}}$ and $K_{\mathrm{DA}} \varepsilon_{\mathrm{C} T}$ in entry 11 with those in entry 8 in Table 3 . Repeated attempts to grow single crystals of the weak yellow complex $\left[\mathrm{MEA}_{2}, \mathbf{C A}\right]$ for $\mathrm{X}$-ray crystallography were unsuccessful. However, from the significantly blue-shifted value of $\lambda_{\mathrm{c}}=$ $452 \mathrm{~nm}$ in column 4, we tentatively conclude that donor-acceptor association of the strong donor $\mathrm{MEA}_{2}$ and chloranil probably occurs at an intermediate interplanar separation of $d$ greater than $3.5 \AA$ (but less than $4.5 \AA$ ). ${ }^{28}$

A close inspection of the charge-transfer absorption of the small and rather powerful $\pi$ acceptor tetracyanoethylene (TCNE) with high concentrations of the highly hindered hexaethylbenzene revealed a weak but distinctive absorbance at $\lambda_{\mathrm{cT}}=540 \mathrm{~nm}$ (Table 4). ${ }^{20} \mathrm{In}$ order to determine how hexaethylbenzene could be subject to partial steric hindrance, we synthesized the hybrid pentaethyltoluene (PET) to establish evidence for donor-acceptor association with TCNE. UV-vis spectral analysis (Figure 3c) indeed revealed a blue complex to be formed in $\mathrm{CH}_{2} \mathrm{Cl}_{2}$ with values of $K_{\mathrm{DA}}$ and $\varepsilon \mathrm{cT}$ which were substantially less than those evaluated for either HMB or TEM. In particular, the value of $\varepsilon_{C T}=300 \mathrm{M}^{-1} \mathrm{~cm}^{-1}$ for the [PET, TCNE] was sharply diminished from $\varepsilon_{\mathrm{CT}}=5200 \mathrm{M}^{-1} \mathrm{~cm}^{-1}$ for [HMB, TCNE], and such a significant difference could result from the reduced m-orbital overlap of PET and TCNE. Although repeated attempts to grow single crystals of [PET, TCNE] suitable for X-ray crystallography failed, QUANTA molecular modeling studies showed that donor-acceptor association was possible at an interplanar separation of $d=3.5 \AA$ by a parallel shift along the aromatic plane, much in the manner previously described in structures $\mathbf{H}$ and $\mathbf{J} .{ }^{24} \mathrm{It}$ is noteworthy that with the exception of the lateral slippage of $\sim 2 \AA$, structure $L$ for [PET, TCNE] is akin to the centrosymmetric structure of [HMB, TCNE] with $d=3.35 \AA$ (established by X-ray crystallography) $)^{29}$ as illustrated in the top perspective $\mathbf{M}$. If so, the donor-acceptor association observed between the highly hindered HEB and tetracyanoethylene, albeit weak (Table 4, entry 4), ${ }^{20}$ may be achieved by a conformational change of a single ethyl group in hexaethylbenzene by a $180^{\circ}$ rotation about the aromatic $-\mathrm{C}_{\alpha}$ bond, ${ }^{21 \mathrm{~b}}$ so as to effect the partial steric hindrance analogous to the [PET, TCNE] structure in L. ${ }^{24}$ Indeed the complete absence of any donor-acceptor association of the conformationally rigid tris-annulated donor TET with tetracyanoethylene (Table 4, entry 5 ) lends a certain credence to this possibility. Be that as it may, the enhanced donor-acceptor association of the tetracyanoethylene with HEB (compared to that with chloranil) was in line with its smaller size and increased acceptor strength. In order to consider these factors further, we next asked how molecular shape could influence the selectivity in donor-acceptor associations by utilizing (a) the powerful diatomic cation $\mathrm{NO}^{+}$with $E_{\text {red }}{ }^{\circ}=1.28 \mathrm{~V}$ vs SCE as well as the uncharged tetranitromethane (TNM) as rather small $\sigma$-acceptors ${ }^{12}$ and (b) the substituted biaryls and a tethered aromatic system to serve as bichromophoric donors, as follows. 


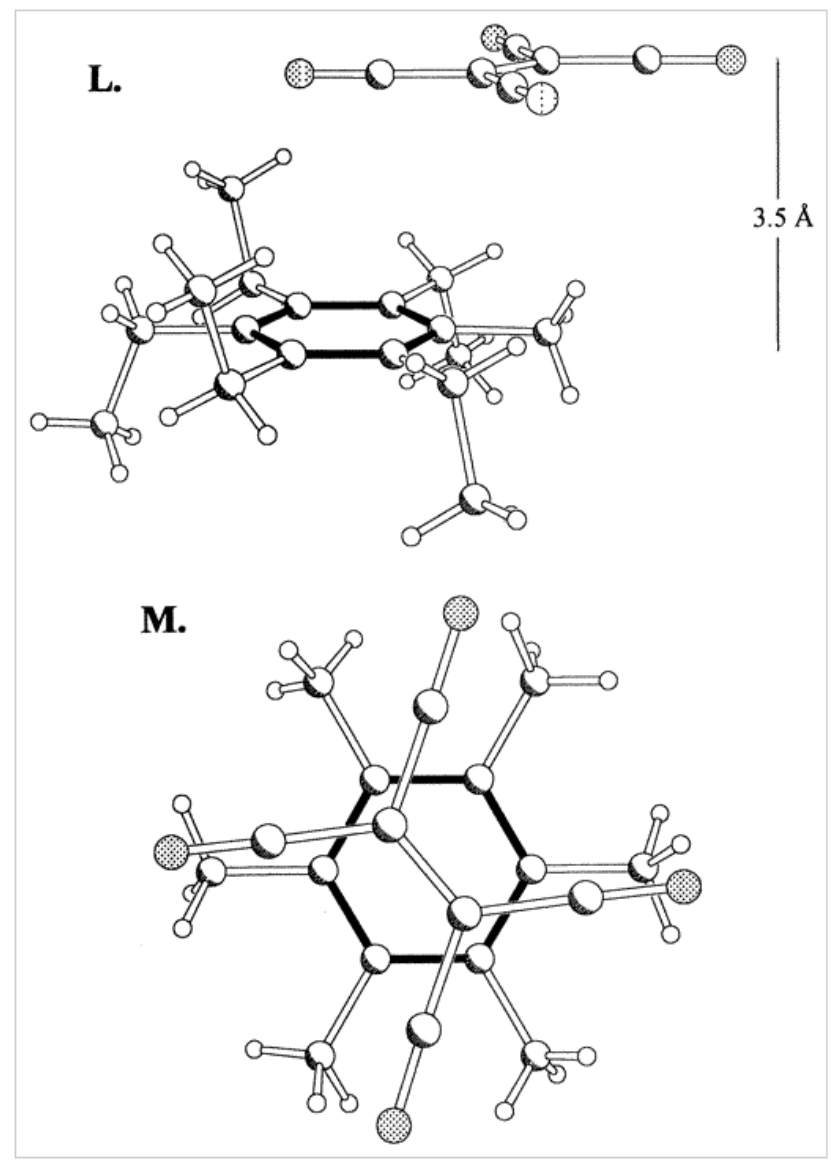

Table 4. Donor-Acceptor Association of Hexaalkylbenzenes with Tetracyanoethylene ${ }^{a}$ 
DONOR $\quad \mathrm{E}_{\text {ox }}^{\text {ox }} \quad \lambda_{\mathrm{CT}} \quad K_{\mathrm{DA}} \quad \varepsilon_{\mathrm{CT}} \quad K_{\mathrm{DA}} \varepsilon_{\mathrm{CT}}$

(V) $(\mathrm{nm}) \quad\left(\mathrm{M}^{-1}\right) \quad\left(\mathrm{M}^{-1} \mathrm{~cm}^{-1}\right)$

$\begin{array}{lllllll}\text { HMB } & 1.62 & 544 & 15.3 & 5,200 & 79,000\end{array}$

$\begin{array}{llllll}\text { TEM } & 1.61 & 542 & 9.81 & 5,000 & 49,000\end{array}$

PET $1.61 \quad 570 \quad 1.91 \quad 300 \quad 600$

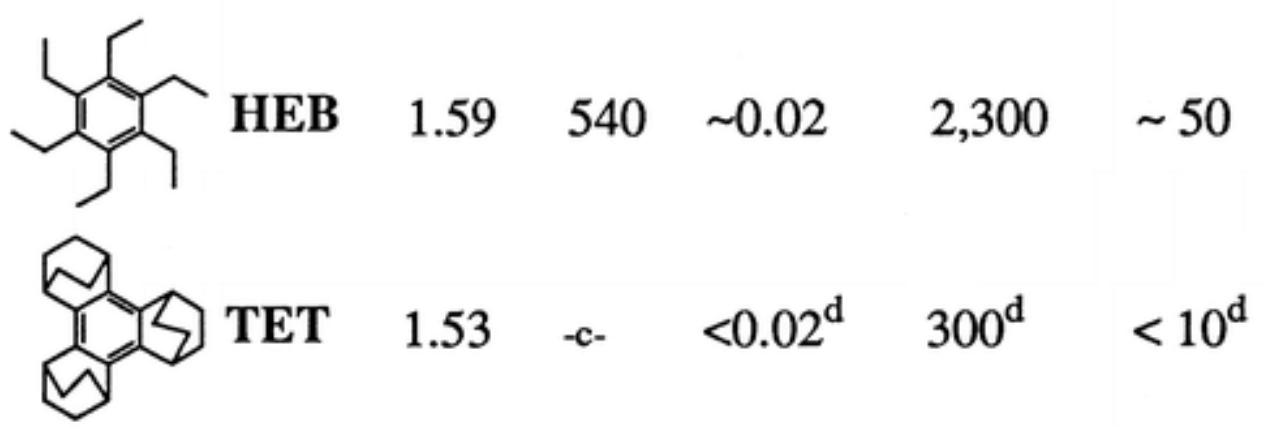

${ }^{a}$ From $2 \mathrm{mM}$ TCNE and $10-100 \mathrm{mM}$ donor in dichloromethane at $24^{\circ} \mathrm{C} .^{c, d}$ See Table 2.

V. Shape Selectivity of Aromatic Donors. A. With $\sigma$-Acceptors. Exposure of nitrosonium $\left(\mathrm{NO}^{+}\right)$tetrafluoroborate to hexamethylbenzene in acetonitrile immediately resulted in an intense red coloration, and the UV-vis spectral analysis of the bright red solution revealed a well-resolved CT absorption band $\left(\lambda_{C T}=337 \mathrm{~nm}\right)$ with a characteristic low-energy band extending beyond $600 \mathrm{~nm}$, as shown in Figure 4A. Although the extinction coefficient of $\varepsilon \mathrm{cT}=$ $3100 \mathrm{M}^{-1} \mathrm{~cm}^{-1}$ in Table 5 was in line with those evaluated for the HMB complexes with the $\pi-$ acceptors in Table 1, the association constant of $K_{\mathrm{DA}}=31000 \mathrm{M}^{-1}$ was more than four orders of magnitude larger - indicative of an exceptionally strong donor-acceptor association of $\mathrm{NO}^{+}$ with HMB. 30 


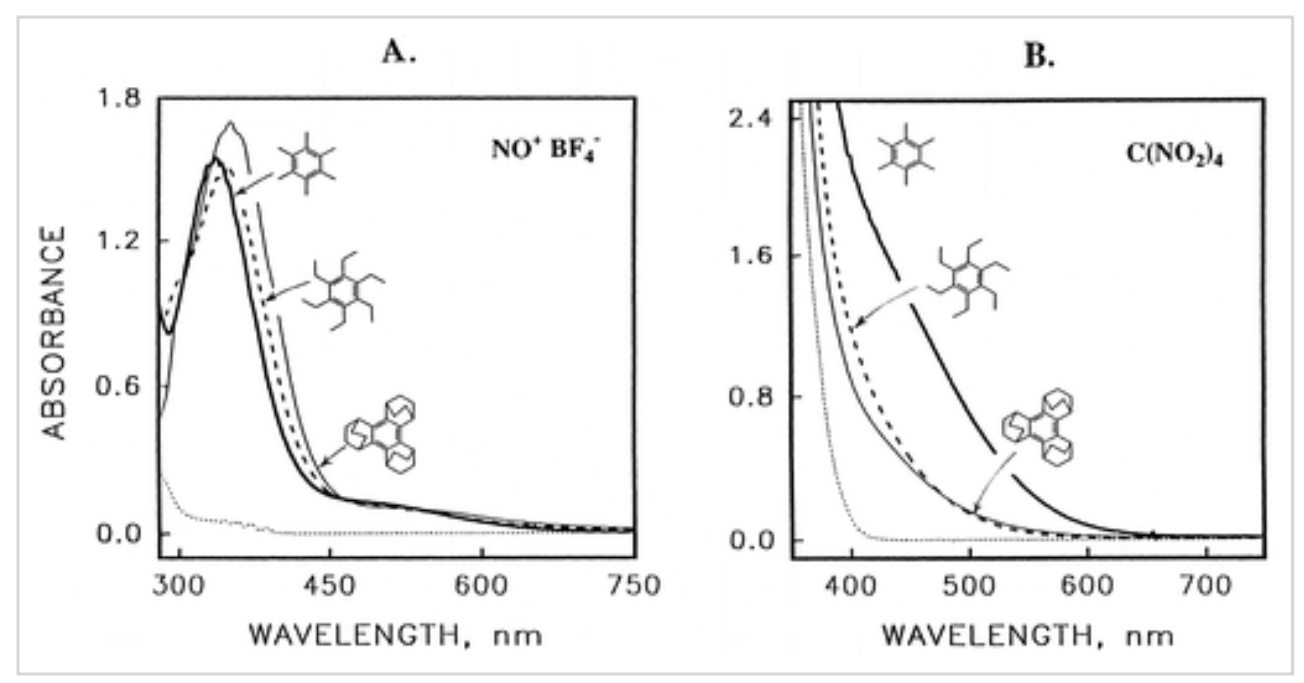

Figure 4 Steric effects in charge-transfer absorptions from $\sigma$-acceptors with aromatic donors: (A) $5 \mathrm{mM} \mathrm{NO}^{+} \mathrm{BF}_{4}^{-}$with $1 \mathrm{mM} \mathrm{HMB}$, HEB, and TET (as indicated); $\mathrm{NO}^{+} \mathrm{BF}_{4}^{-}$alone ( $\cdots$ ) in acetonitrile and (B) 2 M TNM with 5 mM HMB, HEB and TET as indicated; TNM alone ( $\cdots)$ in dichloromethane.

Table 5. Donor-Acceptor Association of Hexaalkylbenzenes with Nitrosonium Tetrafluoroborate ${ }^{a}$

\begin{tabular}{llllcc} 
DONOR & $\mathrm{E}_{\text {ox }}^{\mathrm{o}}$ & $\lambda_{\mathrm{CT}}$ & $K_{\mathrm{DA}}$ & $\varepsilon_{\mathrm{CT}}$ & $K_{\mathrm{DA}} \varepsilon_{\mathrm{CT}}$ \\
& $(\mathrm{V})^{\mathrm{b}}$ & $(\mathrm{nm})$ & $\left(\mathrm{M}^{-1}\right)$ & $\left(\mathrm{M}^{-1} \mathrm{~cm}^{-1}\right)$ \\
\hline
\end{tabular}

\begin{tabular}{lllllll}
\hline HMB & 1.62 & 337 & 31,000 & 3,100 & $9.6 \mathrm{E} 7$
\end{tabular}

$\begin{array}{llllll}\text { HEB } & 1.59 & 347 & 32,500 & 2,900 & 9.4 \mathrm{E} 7\end{array}$<smiles>O=[W]O[Mg]</smiles><smiles>O=[W]O[Mg]</smiles> 
${ }^{a}$ In acetonitrile containing $1 \mathrm{mM} \mathrm{NOBF}_{4}$ and $5-10 \mathrm{mM}$ donor at $24{ }^{\circ} \mathrm{C}$.

Surprisingly, the same red color (with comparable intensity) was observed when hexaethylbenzene was treated with $\mathrm{NO}^{+} \mathrm{BF}_{4}^{-}$under identical (concentration) conditions, and the UV-vis spectrum in Figure 4A confirmed the mostly unaltered charge-transfer absorption. More surprising were the results in Table 5 which showed that the highly hindered trisannulated donors TET and TMT enjoyed undiminished donor-acceptor association with $\mathrm{NO}^{+}$, the measured association constants in all cases being uniformly large, with $K_{\mathrm{DA}}>3 \times 10^{4} \mathrm{M}^{-1}$. In order to account for such an unexpected stability, we grew red crystals of the HEB and TET complexes with $\mathrm{NO}^{+} \mathrm{SbCl}_{6}{ }^{-}$for $\mathrm{X}$-ray crystallographic analysis (see Experimental Section). Indeed, the excellent quality of both single crystals allowed an unambiguous assignment of the nitrogen terminus of the slightly tilted $\mathrm{NO}^{+}$to be centrally situated over the benzenoid (donor) chromophore at a nonbonded distance of $2.08 \AA$ in structures $\mathbf{N}$ and $\mathbf{P}$. The donor-acceptor association, characterized as the very close encounter of $\mathrm{NO}^{+}$to the benzenoid centers of both hexaethylbenzene and TET (inside van der Waals distance), is achieved by significant incursion within the "picket fence" formed by three (alternating) ethyl groups in structure $\mathbf{N}$ and three ethano bridges in structure $\mathbf{P}$. The tight fit of $\mathrm{NO}^{+}$within the van der Waals cavity in the HEB complex (shown in $\mathbf{N}$ ) is sufficient to severely dampen its librational (crystallographic) disorder. ${ }^{31}$ It is particularly noteworthy that such a donor-acceptor interaction derives from the intrinsic donor properties of TET and HEB that are akin to that in the electron-rich HMB, $\underline{33}$ as shown by the constant values of $E_{0 x^{\circ}}$ in Table 4 . As such, the nitrosonium association in the TET and HEB complexes (which occurs in the teeth of the potentially repulsive interactions with the ethyl and ethano substituents) is allowed by the dimensions of the (van der Waals) cavity sufficient to accommodate the diatomic acceptor. ${ }^{34}$ The three-dimensional requirements for the nestling of $\mathrm{NO}^{+}$is graphically illustrated by the space-filling representations $\mathbf{R}$ and $\mathbf{S}$. For comparison, the molecular structure of the unhindered hexamethylbenzene complex ${ }^{32 b}$ is presented in $\mathbf{Q}$.
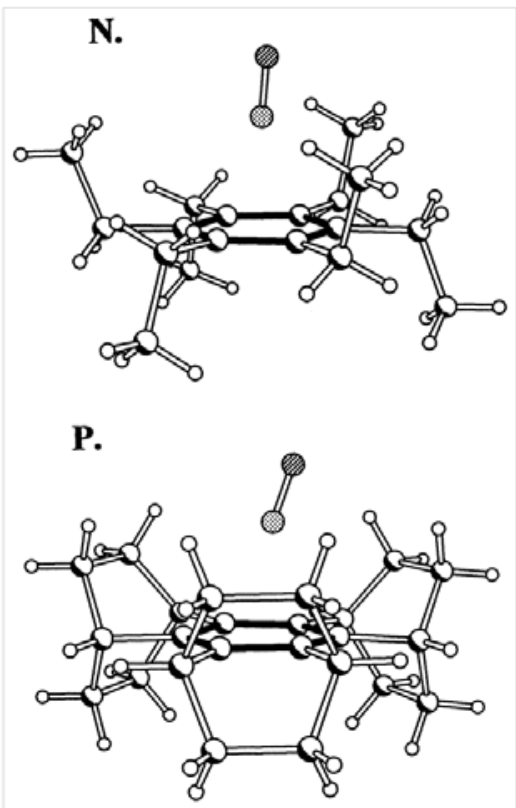


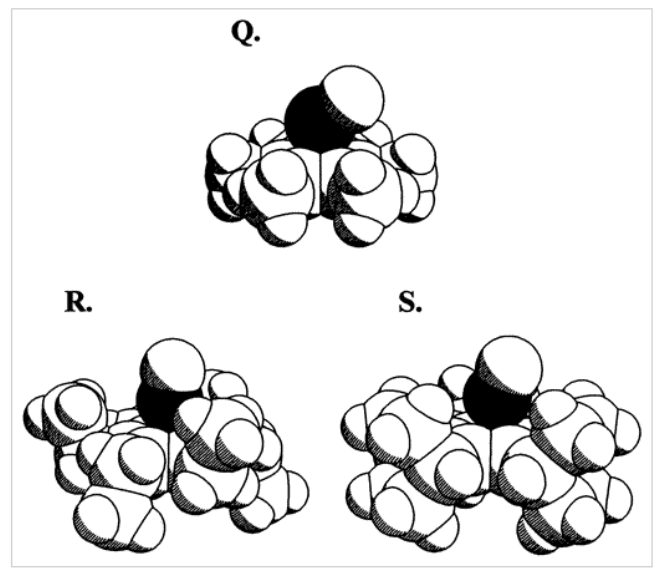

As a further elaboration of the shape-selectivity of aromatic donors, we examined the donor-acceptor interaction with the tetrahedral $\sigma$-acceptor tetranitromethane (TNM). Thus, the exposure of a colorless solution of TNM to hexamethylbenzene in dichloromethane immediately resulted in a dark red solution, the UV-vis spectrum of which showed an intense (unresolved) CT absorption that extended to well beyond $600 \mathrm{~nm}$ (Figure 4B). $\frac{35}{5}$ The quantitative treatment of the absorbance data according to the Benesi-Hildebrand procedure yielded the values of $K_{\mathrm{DA}}$ and $\varepsilon_{\mathrm{C} T}$ for the donor-acceptor association in Table 6 which were substantially less than those for the $\mathrm{NO}^{+}$complex. It is particularly noteworthy that the association of TNM with the hindered HEB resulted in a blue-shift of the nondescript CT tail absorption in Figure 4B, but the quantitative (UV-vis) spectral analysis indicated that the association constant of $K_{\mathrm{DA}}=1.5 \mathrm{M}^{-1}$ was only slightly less than that with $\mathrm{HMB}$ (by about a factor of 3). Furthermore, the highly hindered tris-annulated donors TET and TMT were not significantly different from HEB insofar as their association with TNM. We interpret the rather invariant values of the extinction coefficients in Table 6 (column 5) to be consistent with optimal CT coupling of the small-sized TNM irrespective of steric hindrance from the aromatic donor. Although attempts to grow single crystals of the TNM complexes were unsuccessful, the QUANTA molecular modeling calculations revealed rather large donor-acceptor separations of a single $\mathrm{NO}_{2}$ group of TNM to the HMB centroid in structure $\mathrm{T}$ and to the trisannulated TET in structure $\mathbf{U} . \underline{36}$

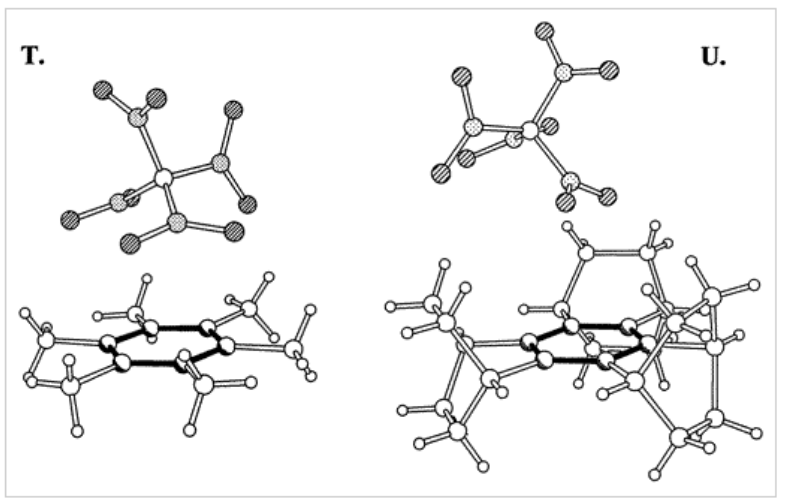


Table 6. Donor-Acceptor Association of Hexaalkylbenzenes with Tetranitromethane ${ }^{a}$

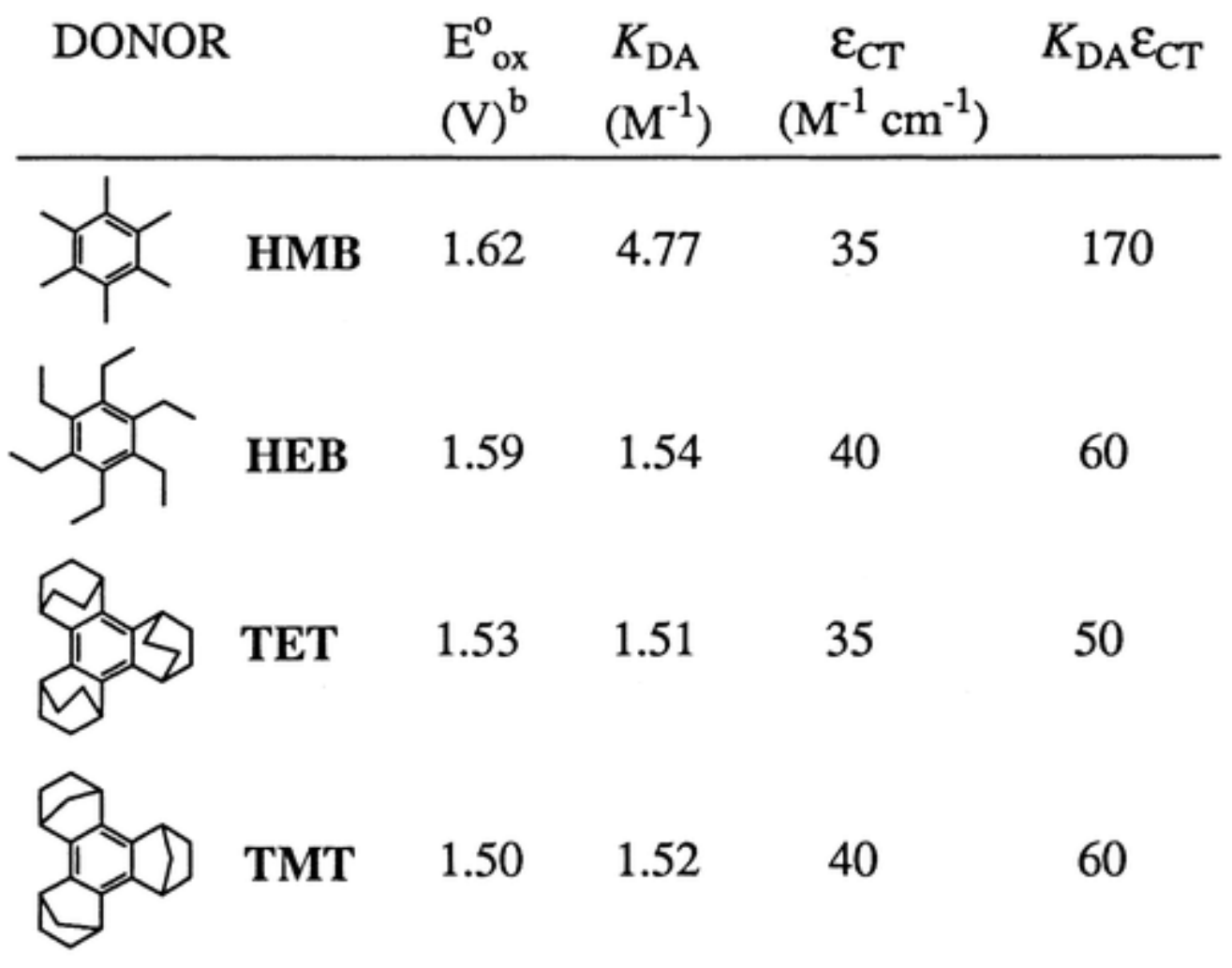

${ }^{a}$ In dichloromethane containing $0.2 \mathrm{M}$ TNM and 5-50 mM donor at $24^{\circ} \mathrm{C}$.

The mixture of hexamethylbenzene with carbon tetrabromide (colorless) resulted in a pale yellow solution which showed a weak tailing UV-vis absorbance without a discernible absorption maximum arising from the blue-shifted charge-transfer band. Such an overlap of the $\mathrm{CT}$ absorption with the local absorption of $\mathrm{CBr}_{4}$ precluded a quantitative evaluation of the donor-acceptor association.

B. In Bichromophoric Systems. Exposure of $p, p^{i}$-dimethylbiphenyl (T-T) to chloranil in dichloromethane was accompanied by an immediate color change to a bright purple solution. UV-vis spectral analysis of the well-resolved CT absorption band $\left(\lambda_{\mathrm{CT}}=502 \mathrm{~nm}\right.$ in Table 7) indicated a very weak association of T-T to chloranil with $K_{\mathrm{DA}}=0.09 \mathrm{M}^{-1}$ and a value of $\varepsilon \mathrm{CT}=$ $6000 \mathrm{M}^{-1} \mathrm{~cm}^{-1}$ in line with the other chloranil complexes with methylbenzenes in Table 1. By analogy with the structure $\mathbf{V}$ (top perspective) of the biphenyl/tetracyanobenzene complex previously established by X-ray crystallography,,$\frac{37}{}$ a similar centrosymmetric structure for the intermolecular association of the bitolyl T-T with chloranil was indicated. In marked contrast, the unsymmetrically permethylated homologue pentamethylphenyl- $p$-toluene (PM-T) afforded a dark purple solution. The UV-vis spectral analysis of the charge-transfer absorption $\left(\lambda_{\mathrm{CT}}=\right.$ $510 \mathrm{~nm}$ ) indicated a substantially larger association with $K_{\mathrm{DA}}=0.99 \mathrm{M}^{-1}$ but significantly diminished value of $\varepsilon_{\mathrm{CT}}=700 \mathrm{M}^{-1} \mathrm{~cm}^{-1}$, which we interpreted as a shape-selective association 
of chloranil with the pentamethylphenyl moiety. Indeed, X-ray crystallography of the purple [PM-T,CA] confirmed this spectral assignment and revealed the interplanar separation of $d=$ $3.41 \AA$ with significant slippage along the pentamethylphenyl plane to avoid the orthogonal tolyl moiety as shown in the side and top perspectives $\mathbf{W}_{\mathbf{s}}$ and $\mathbf{W}_{\mathbf{T}}$.
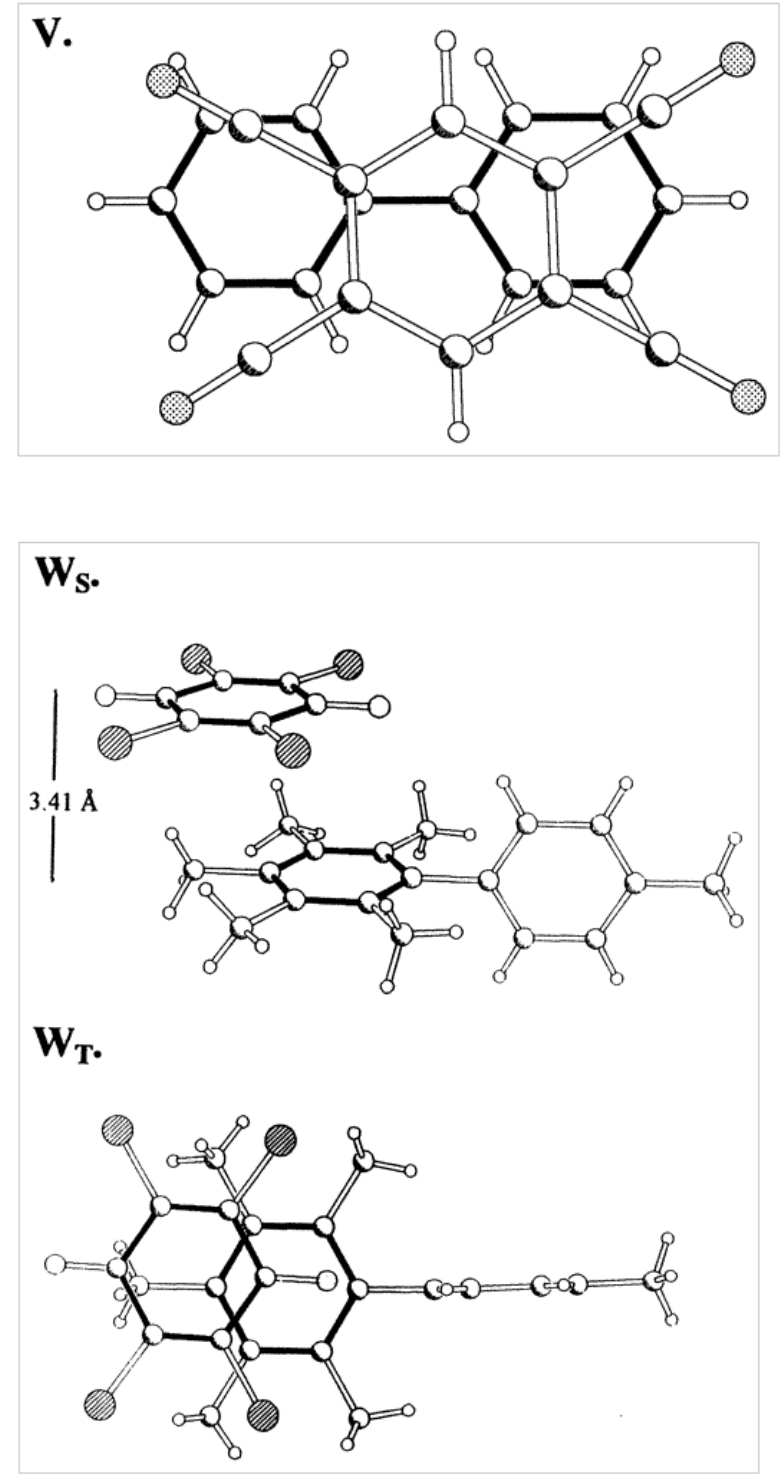

By comparison, the unsymmetrically hindered pentaethylphenyl-p-toluene (PE-T) with chloranil afforded a yellow solution. The charge-transfer absorption $\lambda_{\mathrm{CT}}=437 \mathrm{~nm}$ and $\varepsilon_{\mathrm{CT}}=70 \mathrm{M}^{-1} \mathrm{~cm}^{-1}$ in Table 7 were indicative of an analogous slipped structure for [PE-T,CA], but with the chloranil complexed to the less hindered tolyl moiety. 
Table 7. Donor-Acceptor Association of Various Biphenyls and the Tethered Aromatic Donor with Chloranila

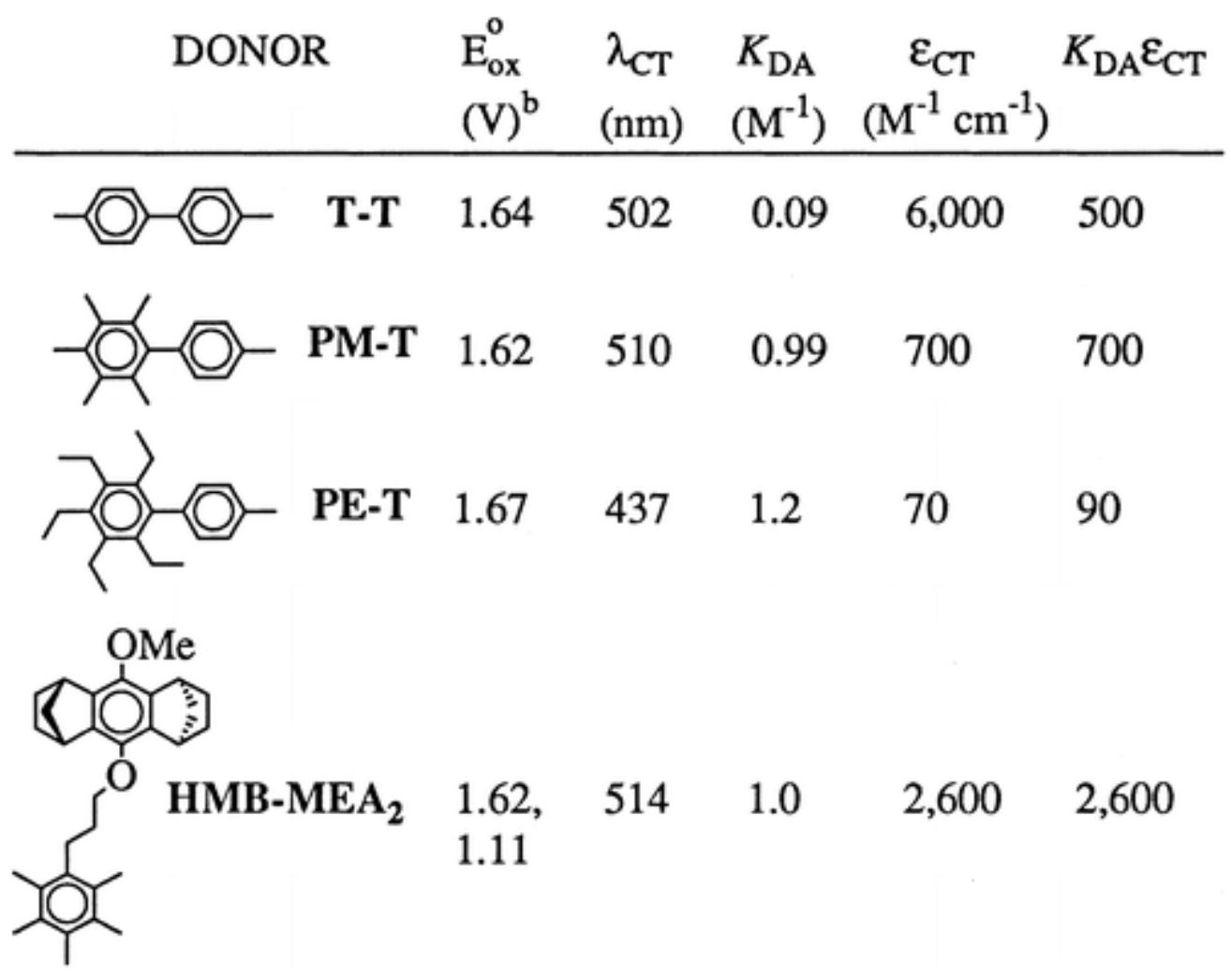

a See Table 1.

A qualitative view of steric hindrance was also useful in predicting the site selectivity of donor-acceptor association in bifunctional aromatic donors that were not directly connected, as in the bichromophoric HMB-MEA 2 in which hexamethylbenzene (HMB) was tethered to the bis-annulated ether MEA2. Thus the treatment of the tethered donor HMB-MEA 2 with chloranil afforded a dark purple solution in which UV-vis spectral analysis yielded the charge-transfer parameters in Table 7 (entry 4) that were essentially identical with those obtained for the mononuclear HMB in Table 1 (entry 1). Indeed, X-ray crystallographic analysis of the [HMB$\mathrm{MEA}_{2}, \mathbf{C A}$ ] complex provided unambiguous support for the predicted structure $\mathbf{Y}$. 


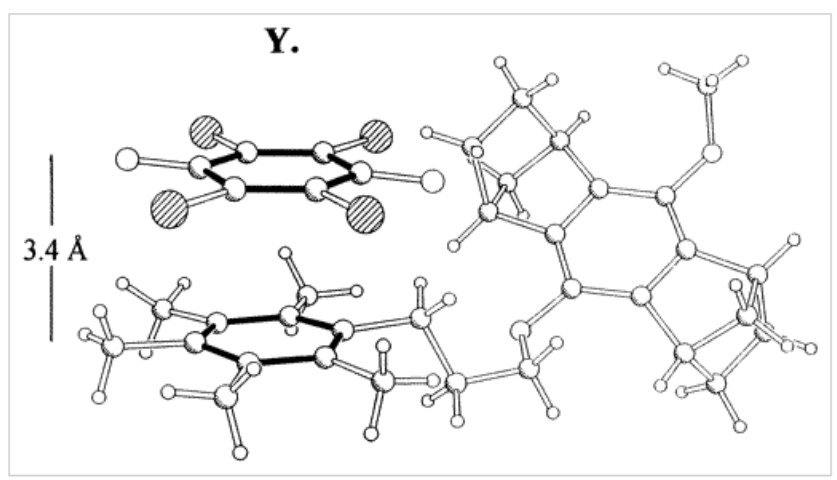

\section{Summary and Conclusions}

Visualization (color) and attendant charge-transfer absorption are reliable and sensitive analytical probes for monitoring the donor-acceptor (DA) association of the various aromatic hydrocarbons in Chart 1 with the different types of $\pi$ - and $\sigma$-acceptors in Chart 2. Thus, the facile molecular association of the polymethylbenzenes, including hexamethylbenzene HMB, with all the $\pi$-acceptors is immediately apparent by the vividly colored solutions, the UV-vis spectral analysis of which reveals new charge-transfer absorption bands $\left(\lambda_{c T}\right)$ for the quantitative evaluation of the association constant $\left(K_{\mathrm{DA}}\right)$ as well as the CT color $\left(\varepsilon_{\mathrm{CT}}\right)$. In marked contrast, the homologous congener hexaethylbenzene HEB shows neither color change nor charge-transfer absorption when exposed to the same m-acceptors under identical conditions. Such unfavored molecular associations of HEB are readily ascribed to its unique conformational structure $\mathbf{D}$, in which the alternant ethyl groups sterically protect both benzenoid faces from the close approach of all planar m-acceptors. Indeed, QUANTA molecular modeling calculations predict the steric envelope around HEB to have an average van der Waals "thickness" of $2 r \simeq 6.4 \AA$, which is significantly larger than that $(4.0 \AA)$ for the lower homologue HMB. As such, we conclude, that the difference of $\Delta r=1.2 \AA$ represents an upper limit for the molecular association of any of the m-acceptors with the benzenoid chromophore.

X-ray crystallography establishes the hexamethylbenzene association with chloranil to be optimized at an interplanar distance of $d=3.51 \AA$ in the cofacial (sandwich) structure $\mathbf{A}$. It is noteworthy that such a donor-acceptor separation corresponds to the sum of van der Waals contact of hexamethylbenzene and chloranil, $\underline{39 a}$ and this conclusion is supported by the $\mathrm{HMB} / \mathrm{CA}$ separation of $r=3.57 \AA$ calculated with the aid of QUANTA molecular modeling. It is tempting to conclude from this computational analysis that molecular associations leading to charge-transfer absorptions derive from inner-sphere interactions involving the intimate van der Waals contact of the donor and acceptor chromophores. ${ }^{39 b}$ According to this formulation, any steric encumbrance of either the donor or acceptor (or both) that extends much beyond the sum of van der Waals radii of the chromophores will lead to sharply diminished chargetransfer absorptions. However, shape selectivity can be an ameliorating factor in at least 2 ways. First, unsymmetrical steric encumbrances in m-donors [such as those in the mono-and di-tert-butyl derivatives TXY and DTT, as well as in semi-annulated TMA and MEA (see Chart 1)] allow the close cofacial (inner-sphere) approach of $\pi$-acceptors by a small parallel shift or 
"slippage" along the aromatic planes, as illustrated by structures $\mathbf{H}, \mathbf{L}$, and $\mathbf{W}$. Second, small electron-poor molecules such as $\sigma$-acceptors [in which the electron deficiency is either largely localized at a single atom as in $\mathrm{NO}^{+}, \mathrm{CBr}_{4}, \mathrm{Br}_{2}$, etc. or on a small group of atoms as in $\mathrm{RNO}_{2}$, $\mathrm{O}_{3}, \mathrm{ArN}_{2}{ }^{+}, \mathrm{SO}_{2}$, etc.] can approach the benzenoid chromophore in sterically encumbered donors [such as hexaethylbenzene HEB and the multiply annulated analogues TET, TMT, OMA, DMA, and MEA ] with van der Waals cavities of sufficient size to allow the nestling of a single (acceptor) center as in the $\mathrm{NO}^{+}$complexes with structures $\mathbf{N}$ and $\mathbf{P}$ or close approach of multiple centers as in the TNM complexes with structures $\mathbf{T}$ and $\mathbf{U}$.

The modulation of steric effects also allows the selective complexation of unsymmetrically substituted biphenyls as in the slipped structure $\mathbf{W}$ and in the tethered bichromophoric donor as in structure $\mathbf{Y}$. The chemical consequences of such shape-selective complexations of (poly)chromic donors and acceptors will be presented separately.

\section{Experimental Section}

Materials. Hexaethylbenzene (Acros), hexamethylbenzene, durene, 4,4'-dimethylbiphenyl, 1,3,5-tri-tert-butylbenzene, and 3,5-di-tert-butyltoluene (Aldrich) were purified by repeated crystallization from ethanol and heptane. Mesitylene (Aldrich) and 1-tert-butyl-3,5dimethylbenzene (Wiley) were purified by fractional distillation. Tetrachloro-p-benzoquinone and tetracyanoethylene (Aldrich) were sublimed in vacuo and recrystallized from benzene. 1,3,5-Trinitrobenzene was purified by crystallization from ethanol. Tropylium tetrafluoroborate (Aldrich) and nitrosonium tetrafluoroborate (Pfaltz and Bauer) were purified by recrystallization from an acetonitrile/dichloromethane mixture. Dimethoxydurene, 40 9,10-dimethoxy1,2,3,4,5,6,7,8-octahydro-1,4:5,8-dimethanoanthracene,, 11 1,2,3,4,5,6,7,8-octahydro-1,4:5,8dimethanoanthracene, $\underline{42}$ 1,4-dimethoxy-2,3-dimethyl-5,6,7,8-tetrahydro-5,8methanonaphthalene: ${ }^{41}$ [oil, ${ }^{1} \mathrm{H}$ NMR $\left(\mathrm{CDCl}_{3}\right) \delta 1.32(\mathrm{~d}, \mathrm{~J}=7.2 \mathrm{~Hz}, 2 \mathrm{H}), 1.54(\mathrm{~d}, \mathrm{~J}=8.7 \mathrm{~Hz}$, $1 \mathrm{H}), 1.76(\mathrm{~d}, \mathrm{~J}=8.7 \mathrm{~Hz}, 1 \mathrm{H}), 2.00(\mathrm{~d}, \mathrm{~J}=7.2 \mathrm{~Hz}, 2 \mathrm{H}), 2.20(\mathrm{~s}, 6 \mathrm{H}), 3.64(\mathrm{~s}, 2 \mathrm{H}), 3.82(\mathrm{~s}, 6 \mathrm{H})$; $\left.{ }^{13} \mathrm{C} \mathrm{NMR}\left(\mathrm{CDCl}_{3}\right) 12.64,27.39,40.89,49.00,61.04,127.53,137.64,148.08\right], 1,1,4,4,5,5,8,8-$ octamethyl-1,2,3,4,5,6,7,8-octahydroanthracene $\frac{43}{3}\left[\mathrm{mp} 222-223^{\circ} \mathrm{C}\right.$ (lit. ${ }^{43} \mathrm{mp} 220-222{ }^{\circ} \mathrm{C}$ ); ${ }^{1} \mathrm{H}$ $\operatorname{NMR}\left(\mathrm{CDCl}_{3}\right) \delta 1.52(\mathrm{~s}, 24 \mathrm{H}), 1.90(\mathrm{~s}, 8 \mathrm{H}), 7.44(\mathrm{~s}, 2 \mathrm{H}) ;{ }^{13} \mathrm{C} \mathrm{NMR}\left(\mathrm{CDCl}_{3}\right) 32.25,34.18$, 35.54], 1,1,4,4-tetramethyl-1,2,3,4,5,6,7,8-octahydroanthracene ${ }^{43}$ [mp 121-122 ${ }^{\circ} \mathrm{C}$ (lit. mp ${ }^{43}$ 121-123 $\left.{ }^{\circ} \mathrm{C}\right) ;{ }^{1} \mathrm{H}$ NMR $\left(\mathrm{CDCl}_{3}\right) \delta 1.38(\mathrm{~s}, 8 \mathrm{H}), 1.71$ (s, 4H), 1.88 (sym m, 4H), 2.84 (sym m, 4H), $7.11(\mathrm{~s}, 2 \mathrm{H}) ;{ }^{13} \mathrm{C} \mathrm{NMR}\left(\mathrm{CDCl}_{3}\right) 26.61,29.30,32.22,34.05,35.44,127.15,134.47$, 142.31], 2,3,4,5,6-pentaethyl-4'-methylbiphenyl $\underline{44}$ [mp 89-91 ${ }^{\circ} \mathrm{C}$; ${ }^{1} \mathrm{H}$ NMR $\left(\mathrm{CDCl}_{3}\right) \delta 1.05$ (t, $\mathrm{J}=$ $7.4 \mathrm{~Hz}, 6 \mathrm{H}), 1.35(\mathrm{t}, J=7.5 \mathrm{~Hz}, 6 \mathrm{H}), 1.38(\mathrm{t}, \mathrm{J}=7.5 \mathrm{~Hz}, 3 \mathrm{H}), 2.44(\mathrm{q}, \mathrm{J}=7.4 \mathrm{~Hz}, 4 \mathrm{H}), 2.52$ (s, $3 \mathrm{H}), 2.82(\mathrm{q}, \mathrm{J}=7.5 \mathrm{~Hz}, 4 \mathrm{H}), 2.85(\mathrm{q}, \mathrm{J}=7.5 \mathrm{~Hz}, 2 \mathrm{H}), 7.24(\mathrm{~d}, \mathrm{~J}=7.8 \mathrm{~Hz}, 2 \mathrm{H}), 7.31(\mathrm{~d}, J=7.8$ $\mathrm{Hz}, 2 \mathrm{H}) ;{ }^{13} \mathrm{C}$ NMR $\left(\mathrm{CDCl}_{3}\right)$ 16.00, 16.10, 21.46, 22.41, 23.79, 128.53, 130.04, 135.72, 137.62, 138.45, 139.36, 139.58, 140.29], and 2,3,4,5,6,4'-hexamethylbipheny $\mid \frac{45}{4}\left[\mathrm{mp} 90-91^{\circ} \mathrm{C} ;{ }^{1} \mathrm{H}\right.$ $\operatorname{NMR}\left(\mathrm{CDCl}_{3}\right) \delta 2.09(\mathrm{~s}, 6 \mathrm{H}), 2.40(\mathrm{~s}, 6 \mathrm{H}), 2.44(\mathrm{~s}, 3 \mathrm{H}), 2.54(\mathrm{~s}, 3 \mathrm{H}), 7.14(\mathrm{~d}, \mathrm{~J}=7.8 \mathrm{~Hz}, 2 \mathrm{H})$, 7.35 (d, $\mathrm{J}=7.8 \mathrm{~Hz}, 2 \mathrm{H}) ;{ }^{13} \mathrm{C} \mathrm{NMR}\left(\mathrm{CDCl}_{3}\right)$ 16.82, 16.98, 18.59, 21.41, 129.14, 129.57, 131.00, 132.45, 134.00, 135.81, 139.99, 140.18], 1,2,3,4,5,6,7,8,9,10,11,12-dodecahydro-1,4:5,8:9,12triethanotriphenylene ${ }^{46}$ (TET), and 1,2,3,4,5,6,7,8,9,10,11,12-dodecahydro-1,4:5,8:9,12trimethanotriphenylene ${ }^{47}$ (TMT) were available from literature procedures. Synthesis of the 
tethered 9-[3-(pentamethyl-phenyl)-1-propyloxy]-10-methoxy-1,2,3,4,5,6,7,8-octahydro-1,4:5,8dimethanoanthracene (HEB-MEA 2 ) will be described elsewhere. Pentaethyltoluene (PET) was prepared from hexaethylbenzene by refluxing a mixture of hexaethylbenzene $(6.15 \mathrm{~g}, 25$ $\mathrm{mmol})$ and acetyl chloride $(2.0 \mathrm{~g}, 25.5 \mathrm{mmol})$ in carbon disulfide $(25 \mathrm{~mL})$ in the presence of anhydrous aluminum chloride for $8 \mathrm{~h}$. The resulting deep brown solution was cooled to room temperature, poured over a mixture of ice $(200 \mathrm{~g})$, concentrated hydrochloric acid $(25 \mathrm{~mL})$, and extracted with ether $(4 \times 50 \mathrm{~mL})$. The combined ether extracts were washed with water and dried over anhydrous magnesium sulfate. Removal of the solvent and recrystallization form ethanol afforded white needles of pentaethylacetophenone $\frac{48}{2}(6.2 \mathrm{~g}, 95 \%)$; mp $136-137^{\circ} \mathrm{C}$ (lit. $\left.{ }^{48} \mathrm{mp} 136-137^{\circ} \mathrm{C}\right)$. A solution of pentaethylacetophenone $(6.0 \mathrm{~g}, 23 \mathrm{mmol})$ in trifluoroacetic acid $(25.5 \mathrm{~mL})$ and water $(4.5 \mathrm{~mL})$ was refluxed for $16 \mathrm{~h}$ and cooled to room temperature. The dark brown reaction mixture was poured over ice and extracted with ether $(4 \times 50 \mathrm{~mL})$. The ether layers were washed with water and dried over anhydrous magnesium sulfate.

Evaporation of the solvent in vacuo furnished pentaethylbenzene as an oil [GC-MS $\mathrm{m} / \mathrm{z} 218$ $\left(\mathrm{M}^{+}\right)$, calcd for $\mathrm{C}_{16} \mathrm{H}_{26}$ ]. The resulting crude pentaethylbenzene was dissolved in acetic acid (15 $\mathrm{mL}$ ) and treated with paraformaldehyde $(0.75 \mathrm{~g}, 25 \mathrm{mmol})$ and $5 \mathrm{~mL}$ of a $31 \mathrm{wt} \% \mathrm{HBr}$ in acetic acid. ${ }^{49}$ The mixture was heated for $12 \mathrm{~h}$ at $80^{\circ} \mathrm{C}$ and then poured into $100 \mathrm{~mL}$ water. The crystalline precipitate thus formed was filtered off, washed with water, and dried in vacuo to afford (bromomethyl)pentaethylbenzene $(7.0 \mathrm{~g}, 22.5 \mathrm{mmol})$; GC-MS m/z 310, $312\left(\mathrm{M}^{+}\right)$, calcd for $\mathrm{C}_{17} \mathrm{H}_{27} \mathrm{Br}$. To a solution of crude (bromomethyl)pentaethylbenzene $(6.22 \mathrm{~g}, 20 \mathrm{mmol})$ in anhydrous diethyl ether $(100 \mathrm{~mL})$ was added lithium aluminum hydride $(0.76 \mathrm{~g}, 20 \mathrm{mmol})$, and the mixture was refluxed for $2 \mathrm{~h}$. The usual aqueous workup resulted an oily residue which upon crystallization form ethanol afforded pure pentaethyltoluene as colorless prisms $(3.7 \mathrm{~g}$, 80\%): $\mathrm{mp} \mathrm{45-46}{ }^{\circ} \mathrm{C} ;{ }^{1} \mathrm{H} \mathrm{NMR}\left(\mathrm{CDCl}_{3}\right) \delta 1.44$ (sym m, $\left.15 \mathrm{H}\right), 2.54(\mathrm{~s}, 3 \mathrm{H}), 2.93$ (quintet, 10H); ${ }^{13} \mathrm{C} \mathrm{NMR} \mathrm{( \textrm {CDCl } _ { 3 } )}$ 14.86, 15.38, 15.91, 16.07, 22.24, 22.51, 23.16, 132.18, 137.71, 138.26, 138.62. GC-MS m/z $\left(\mathrm{M}^{+}\right)$, 232, calcd for $\mathrm{C}_{17} \mathrm{H}_{28}$. Anal. Calcd for $\mathrm{C}_{17} \mathrm{H}_{28}: \mathrm{C}, 87.86 ; \mathrm{H}, 12.14$. Found: C, 88.06; $\mathrm{H}, 12.00$. Triethylmesitylene (TEM) was prepared by dropwise addition of a $30 \%$ solution of $\mathrm{HBr}$ in acetic acid $(100 \mathrm{~mL})$ to a stirred mixture of paraformaldehyde $(12 \mathrm{~g}, 0.4$ $\mathrm{mol})$ and 1,3,5-triethylbenzene $(16.2 \mathrm{~g}, 0.1 \mathrm{~mol})$ in glacial acetic acid $(25 \mathrm{~mL})$ at $25^{\circ} \mathrm{C} .{ }^{49}$ The resulting mixture was heated at $100^{\circ} \mathrm{C}$ for $72 \mathrm{~h}$, cooled to room temperature, and poured over ice-water $(250 \mathrm{~mL})$ mixture. The precipitate thus obtained was filtered, washed with water, and dried in vacuo. The crude pale brown solid $(38 \mathrm{~g})$ was dissolved in diethyl ether $(200 \mathrm{~mL})$ and added dropwise to a suspension of lithium aluminum hydride $(10 \mathrm{~g})$ in diethyl ether during a 1 $\mathrm{h}$ period. The resulting mixture was refluxed for $4 \mathrm{~h}$ and cooled to room temperature. The usual workup as above and crystallization from ethanol afforded pure triethylmesitylene (74\%): $\mathrm{mp}$ $55-56{ }^{\circ} \mathrm{C}$; ${ }^{1} \mathrm{H} \mathrm{NMR}\left(\mathrm{CDCl}_{3}\right) \delta 1.22(\mathrm{t}, \mathrm{J}=7.5 \mathrm{~Hz}, 9 \mathrm{H}), 2.37(\mathrm{~s}, 9 \mathrm{H}), 2.79$ (q, J = 7.5 Hz, 6H); ${ }^{13} \mathrm{C} \mathrm{NMR}\left(\mathrm{CDCl}_{3}\right)$ 13.97, 15.50, 23.79, 131.43, 138.88. GC-MS m/z $\left(\mathrm{M}^{+}\right), 204$, calcd for $\mathrm{C}_{15} \mathrm{H}_{24}$. Anal. Calcd for $\mathrm{C}_{15} \mathrm{H}_{24}$ : C, 88.16; $\mathrm{H}, 11.84$. Found: C, 88.37; $\mathrm{H}, 11.68$.

Dichloromethane (Mallinckrodt analytical reagent) was repeatedly stirred with fresh aliquots of concentrated sulfuric acid ( $20 \%$ by volume) until the acid layer remained clear. After separation, it was washed successively with water, aqueous sodium bicarbonate, water, and aqueous sodium chloride and dried over anhydrous calcium chloride. The dichloromethane was distilled twice from $\mathrm{P}_{2} \mathrm{O}_{5}$ under an argon atmosphere and stored in a Schlenk tube 
equipped with a Teflon valve fitted with Viton O-rings. Acetonitrile (Fischer) was stirred with $\mathrm{KMnO}_{4}$ for $24 \mathrm{~h}$, and the mixture was refluxed until the liquid was colorless. The $\mathrm{MnO}_{2}$ was removed by filtration. The acetonitrile was distilled from $\mathrm{P}_{2} \mathrm{O}_{5}$ under an argon atmosphere and then refluxed over $\mathrm{CaH}_{2}$ for $6 \mathrm{~h}$. After distillation from the $\mathrm{CaH}_{2}$, the solvent was stored in a Schlenk flask under an argon atmosphere. The UV-vis absorption spectra were recorded on a Hewlett-Packard 8450A diode-array spectrometer. The ${ }^{1} \mathrm{H}$ and ${ }^{13} \mathrm{C}$ NMR spectra were recorded on a General Electric QE-300 spectrometer, and chemical shifts are reported in ppm units downfield from tetramethylsilane.

The Formation Constants of Donor-Acceptor Complexes. General Procedure. Typically, in a 1-cm square quartz cuvette (UV cell) equipped with a side arm and Schlenk adapter was placed 1-4 mM solution of chloranil (with the aid of a hypodermic syringe) under an argon atmosphere. A known amount of arene donor was added in increments, and the absorbance changes were measured at the absorption maxima as well as two other wavelength close to the absorption maxima (see Figure 1). The absorbance data were then evaluated with the aid of the Benesi-Hildebrand correlation in eq 2. ${ }^{16}$ From the linear plot of [CA]/Act against [arene] ${ }^{-}$ ${ }^{1}$, consisting of at least eight data points, the slope was estimated as $\left(K_{D A \varepsilon C T}\right)^{-1}$ and the intercept as $(\varepsilon c T)^{-1}$. Linear fits obtained by least square method had a correlation coefficient of $>0.999$. The runs were made in duplicate to ensure the reproducibility of the spectra. Detailed procedures for the quantification of DA complex formation with various electron acceptors such as tetracyanoethylene, ${ }^{18} 1,3,5$-trinitrobenzene, ${ }^{11}$ tropylium tetrafluoroborate, $\underline{50}$ tetranitromethane, ${ }^{35}$ and nitrosonium tetrafluroborate ${ }^{30}$ have been described previously. Estimation of the Formation Constants for DA Complexes from Hindered Donors. In cases of the hindered arene donors (in which no charge-transfer absorption band was detected), the upper limits of the formation constants (KDA) were estimated as follows. The detection limit of the spectrophotometer was taken as 0.01 absorbance unit for an average charge-transfer extinction coefficient of $\varepsilon_{\mathrm{CT}}=1000 \mathrm{M}^{-1} \mathrm{~cm}^{-1}$. We calculated the lowest concentration of the donor-acceptor complex detectable by UV-vis spectroscopy to be $[D, A]=$ $0.01 / \varepsilon_{\mathrm{CT}}=1 \times 10^{-5} \mathrm{M}$. If no DA complex was detected at a donor concentration of $[D]=0.1-0.5$ $\mathrm{M}$ and an acceptor concentration of $[\mathrm{A}]=4-10 \mathrm{mM}$, the upper limit for the formation constant was taken as $K_{\mathrm{DA}}=[\mathrm{DA}] /[\mathrm{D}][\mathrm{A}]<0.025-0.002 \mathrm{M}^{-1}$ (see Tables 1 and 2). The DA complexes with $K_{\mathrm{DA}}<0.025 \mathrm{M}^{-1}$ were generally considered to be contact charge-transfer complexes. $\frac{8,51}{.}$

\section{Simulation of the Intermolecular Separations in Donor-Acceptor Complexes by} Molecular Modeling Calculations. The most energetically favorable separation of the donor and acceptor in isolated $[D, A]$ complexes was searched with the aid of molecular modeling calculations (CHARMm program, ${ }^{54}$ vers. 22, Molecular Simulation Inc., 1994) which included both intramolecular force field (such as covalent bond, bond angle, and torsion angle potentials) and intermolecular interactions (including Columbic and Lennard-Jones potential terms). The initial molecular models of the interacting arene donors and the various electron acceptors (see Charts 1 and 2) were constructed with the aid of the graphics package QUANTA ${ }^{15}$ running on a Silicon Graphics ONYX Reality workstation. The optimization of the geometry of the individual molecules was carried out by initial energy minimization using the Steepest Descents (SD) algorithm ${ }^{53}$ to remove any obvious improper conformations, and this 
was followed by 1000 steps of ABNR $\underline{54}$ (Adopted Basis of Newton-Raphson) algorithm in "CHARMm" to reach the minimum of molecular potential energy. The charges of the individual molecules were balanced using Gasteiger's method, ${ }^{52}$ thereby resulting in neutral molecules. The energy minimization package CHARMm allowed any number of molecules within a single structure file to be simulated. Thus, the optimized donor and acceptor molecules were put together in random orientation and the intermolecular separation distance between them was varied from 3.0 to $5.0 \AA$, and the relative orientation of the interacting molecules was also varied prior to the energy minimization. The energy minimization of the various resulting aggregates was carried out in a similar way as described for the individual molecules, i.e., using the Steepest Descents algorithm ${ }^{53}$ to remove unfavorable steric contacts followed by 1000 steps of $A B N R^{54}$ algorithm. The geometry and the separation distances between donor and acceptors molecules of the minimized structures were analyzed using the XP graphical package $\underline{56}$ after the energy tolerance was satisfied.

Isolation and X-ray Crystallography of Donor-Acceptor Complexes. The chloranil complexes were crystallized from an equimolar solution of CA and HMB, TEM, or PM-T by very slow evaporation of dichloromethane. Since the similar treatment of the tethered HEBMEA 2 yielded an amorphous solid, a $1: 1 \mathrm{CH}_{2} \mathrm{Cl}_{2} / \mathrm{CCl}_{4}$ mixture was used to prepare dark purple crystals of [HMB-MEA $2, \mathrm{CA}$ ]. For the nitrosonium complexes, hexaethylbenzene (HEB) was added to a flask that contained nitrosonium hexachloroantimonate, and a minimum amount of dichloromethane was added under an argon atmosphere at $0{ }^{\circ} \mathrm{C}$. The undissolved solid was removed by filtration, and the resulting dark colored solution was carefully layered with anhydrous toluene $(30 \mathrm{~mL})$ and stored in the refrigerator $\left(-10^{\circ} \mathrm{C}\right)$. After $48-72 \mathrm{~h}$, dark red crystals of $\mathrm{HEB} / \mathrm{NO}^{+}$complex were deposited at the dichloromethane/toluene interface. A similar procedure yielded dark red crystals of the TET/NO ${ }^{+}$complex.

The X-ray crystallography of the donor-acceptor complexes was carried out with a Siemens SMART diffractometer equipped with a CCD detector at $-150^{\circ} \mathrm{C}$. The structures were solved by direct methods ${ }^{55}$ and refined with IBM Pentium and SGI Indigo computers. In the various chloranil complexes, the intramolecular dimensions of CA and the arene donors were the same as those observed in uncomplexed molecules. However, the arene complexed to nitrosonium cation in $\left[\mathrm{HEB}, \mathrm{NO}^{+}\right]$and $\left[\mathrm{TET}, \mathrm{NO}^{+}\right]$showed slight lengthening of all aromatic $\mathrm{C}=$ $\mathrm{C}$ double bonds when compared with crystal structures of the uncomplexed donors. The pertinent intermolecular orientations as PLUTO plots (generated from the XP graphical package $)^{56}$ are shown in structures $\mathbf{A}, \mathbf{C}, \mathbf{N}, \mathbf{P}, \mathbf{W}$, and $\mathbf{Y}$, and the critical interplanar separations (d) are included. The crystallographic data are on deposit and can be obtained from the Director, Cambridge Crystallographic Data Centre, 12 Union Road, Cambridge, CB2 1EZ, U.K.

\section{Acknowledgment}

We thank Professor B. M. Pettitt and Dr. R. Mitra (Institute for Molecular Design, University of Houston) and M.S.I. for permission and invaluable help with the molecular modeling 
calculations and the National Science Foundation and R. A. Welch Foundation for financial support.

\section{Supporting Information Available}

Tables of crystal structure data for [TEM,CA], [PM-T,CA], [HMB-MEA $2, \mathrm{CA}$ ], [HEB, $\mathrm{NO}^{+} \mathrm{SbCl}_{6}^{-}$], and $\left[\mathrm{TET}, \mathrm{NO}^{+} \mathrm{SbCl}_{6}^{-}\right]$including atomic coordinates, anisotropic thermal parameter, bond lengths, and bond angles and ORTEP diagrams showing thermal ellipsoids (36 pages). See any current masthead page for ordering and Internet access instructions.

PDF ja9393.pdf (1.1 MB)

\section{References}

1 (a) Meissner, R. S.; Rebek, J., Jr.; de Mendoza, J. Science 1995, 270, 1485. (b) Grotzfeld, R. M.; Branda, N.; Rebek, J., Jr. Science 1996, 271, 487. (c) Wintner, E. A.; Rebek, J., Jr. Acta Chem. Scand. 1996, 50, 469 and references therein. (d) Mascal, M. Contemp. Org. Syn. 1994, 1, 1. (e) Lawrence, D. S.; Jiang, T.; Levett, M. Chem. Rev. 1995, 95, 2229. (f) Still, C. W. Acc. Chem. Res. 1996, 29, 155. (g) Hartshorn, C. M.; Steel, P. J. J. Chem. Soc., Chem. Commun. 1997, 541.

$\underline{2}$ (a) Cram, D. J. Nature 1992, 356, 29. (b) Cram, D. J. Angew. Chem., Int. Ed. Engl. 1988, 27, 1009 and references therein. (c) Lehn, J.-M. Angew. Chem., Int. Ed. Engl. 1988, 27, 89 and references therein. (d) Lehn, J.-M. Pure Appl. Chem. 1994, 66, 1961. (e) Hunter, C. A. Angew. Chem., Int. Ed. Engl. 1995, 34, 1679. (f) Metzger, A.; Lynch, V. M.; Anslyn, E. V. Angew. Chem., Int. Ed. Engl. 1997, 36, 862.

$\underline{3}$ (a) Philp, D.; Stoddart, J. F. Angew. Chem., Int. Ed. Engl. 1996, 35, 1154. (b) Balzani, V. Tetrahedron 1992, 48, 10433. (c) Stang, P. J.; Olenyuk, B. Angew. Chem., Int. Ed. Engl. 1996, 35, 732. For donor-acceptor interactions in the solid state for crystal engineering, see: Desiraju, G. R. Angew. Chem., Int. Ed. Engl. 1995, 34, 2311.

4 (a) Mulliken, R. S. J. Am. Chem. Soc. 1952, 74, 811. (b) Mulliken, R. S.; Person, W. B. Molecular Complexes. A Lecture and Reprint Volume; Wiley: New York 1969. (c) Foster, R. Organic Charge-Transfer Complexes; Academic: New York, 1969. (d) Briegleb, G. Elektronen - Donator-Acceptor Komplexe; Springer: Berlin, 1961.

$\underline{5}$ (a) The charge-transfer absorption $\left(\lambda_{c T}\right)$ generally occurs in the UV-vis region with $h c / \lambda_{c T}=$ IP - EA - $\omega,{ }^{4}$ where IP is the ionization potential of the electron donor (D), EA is the electron affinity of the electron acceptor $(A)$, and $\omega$ is the electrostatic energy of the ion pair $\left[D^{*+}, A^{*}\right]$. (b) To first approximation, the separation $d$ in the DA complex is directly related to $\omega$, i.e., $d=e^{2} / \omega$ which is especially applicable to planar donors and acceptors.

$\underline{6}$ Charge-transfer interactions have been employed in the design/synthesis of self-assembling catenanes, rotaxanes, and other molecular machines. See: (a) Bissell, R. A.; Córdova, E.; Kaifer, A. E.; Stoddart, J. F. Nature 1994, 369, 133. (b) Córdova, E.; Bissell, R. E.; Kaifer, A. E. J. Org. Chem. 1995, 60, 1033. (c) Asakawa, M.; Ashton, P. R.; Boyd, S. E.; Brown, C. L.; Gillard, R. E.; Kocian, O.; Raymo, F. M.; Stoddart, J. F.; Tolley, M. S.; White, A. J. P.; Williams, D. J. J. Org. Chem. 1997, 62, 26. (d) Toki, A.; Yonemura, H.; Matsuo, T. Bull. Chem. Soc. Jpn. 1993, 66, 3382.

$\underline{7}$ For example, the highly colored trinitrobenzene/arene complexes have found extensive use in the purification and characterization of aromatic hydrocarbons owing to their ready 
crystallization with definite stoichiometry and sharp melting points. See: Weiss, J. J. Chem. Soc. 1942, 245. Powell, H. M.; Huse G.; Cook, P. W. J. Chem. Soc. 1943, 153. Parini, V. P. Russ. Chem. Rev. 1962, 31, 408.

8 The transitory contact charge-transfer complexes have been described by: Tamres, M.; Strong, R. L. In Molecular Association; Foster, R. F., Ed.; Academic: New York, 1979; Vol. 2, p 331ff.

$\underline{9}$ (a) There are a few cases mentioned in the literature where electron-rich but sterically congested donors such as hexaisopropylbenzene (Arnett, E. M.; Bollinger, J. M. J. Am. Chem. Soc. 1964, 86, 4729), hexacyclopropylbenzene (Usieli, V.; Victor, R.; Sarel, S. Tetrahedron Lett. 1976, 2705), and 1,4:5,8:9,12-triethanododecahydrotriphenylene (Komatsu, K.; Aonuma, S.; Jimbu, Y.; Tsuji, R.; Hirosawa, C.; Takeuchi, K. J. Org. Chem. 1991, 56, 195), etc. show no charge-transfer absorptions with such commonly used electron acceptors as tetracyanoethylene (see: Merrifield, R. E.; Phillips, W. D. J. Am. Chem. Soc. 1950, 80, 2778 and Foster, R. in ref 4c, p 197). However, there is no systematic study extant in the literature to describe steric effects on donor-acceptor complexation.

10 As evaluated by the ionization potentials (IP, gas phase) and oxidation potentials ( $E_{\mathrm{ox}}{ }^{\circ}$, solution) of benzenoid hydrocarbons, which are mostly dependent on the number and position of carbon-centered substituents, see: Howell, J. O.; Goncalves, J. M.; Amatore, C.; Klasinc, L.; Wightman, R. M.; Kochi, J. K. J. Am. Chem. Soc. 1984, 106, 3968. For the relevant values of IP and $E_{0 x^{\circ}}$ of the other donors in Chart 1, see Tables 2 and 3.

11 The acceptor strengths in Chart 2 can be conveniently evaluated by the reduction potentials. ${ }^{4}$ For TCNE, Ered ${ }^{\circ}=+0.24$ V: Rehm, D.; Weller, A. Isr. J. Chem. 1970, 8, 259. Tropylium, $E_{r e d}{ }^{\circ}=-0.18$ V: Wasielweski, M. R.; Breslow, R. J. Am. Chem. Soc. 1976, 98, 4222. Chloranil, $E_{\text {red }}^{\circ}=0.02$ V: Mann, C. K.; Barnes, K. K. Electrochemical Reactions in Nonaqueous Systems; Dekker: New York, 1970. Trinitrobenzene, Ered $^{\circ}=$ -0.42: Körtüm, G.; Walz, H. Z. Electrochem. 1955, 59, 184.

12 For nitrosonium, $E_{\text {red }^{\circ}}=+1.28 \mathrm{~V}$ : Lee, K. Y.; Kuchynka, D. J.; Kochi, J. K. Inorg. Chem. 1990, 29, 4196; carbon tetrabromide, $E_{\text {red }}{ }^{\circ}=-0.30$ V: Stackelberg, M.; Stracke, W. Z. Electrochem. 1949, 53, 118. Also, see: Al-Ekabi, H.; Draper, A. M.; de Mayo, P. Can. J. Chem. 1989, 69, 1061. Tetranitromethane, $E_{\text {red }}{ }^{\circ}=0.00$ V: Altukhov, K. V.; Perekalin, V. V. Russ. Chem. Rev. 1976, 45, 1052. Bromine, $E_{\text {red }}{ }^{\circ}$ is unmeasured.

13 Harding, T. T.; Wallwork, S. C. Acta Crystallogr. 1955, 8, 787. Also, see: Jones, N. D.; Marsh, R. E. Acta Crystallogr. 1962, 15, 809.

14 Molecular structures presented hereinafter as PLUTO plots (Motherwell, W. D. S.; Clegg, W. Program for Plotting Molecular and Crystal Structures 1978, Cambridge, U. K.) were produced with the aid of an XP-graphical package. ${ }^{56}$

15 QUANTA (vers. 4.11) from Molecular Simulations, Inc., 16 New England Executive Park, Burlington, MA 081803-5297. See: Experimental Section for a brief description of the molecular modeling package.

16 (a) Benesi, H. A.; Hildebrand, J. J. J. Am. Chem. Soc. 1949, 71, 2703. (b) Person, W. B. J. Am. Chem. Soc. 1965, 87, 167. (c) Foster, R. Molecular Complexes; Crane, Russak \& Co.: New York, 1974; Vol. 2.

17 (a) Horner, L.; Merz, H. Ann. Chem. 1950, 89, 570. (b) Rathore, R.; Kochi, J. K. Tetrahedron Lett, 1994, 35, 8577. (c) Bockman, T. M.; Perrier, S.; Kochi, J. K. J. Chem. 
Soc., Perkin Trans. 2 1993, 595. (d) Reichenback, G.; Santini, S.; Mazzucato, U. J. Chem. Soc., Faraday Trans. 1 1973, 49, 143.

18 The slope of the Benesi-Hildebrand relationship as given by $K_{\mathrm{DAECT}}$ is the "effective absorbance", see: Frey, J. E.; Andrews, A. M.; Ankoviac, G. G.; Beaman, D. N.; DuPont, L. E.; Elsner, T. E.; Lang, S. R.; Zwart, M. A. O.; Seagle, R. E.; Torreano, L. A. J. Org. Chem. 1990, 55, 606.

19 For the tetracyanoethylene data, see: Table 4.

20 The values of $K_{\mathrm{DAECT}}$ in the table provide a numerical guide to the visual intensity of the charge-transfer colors. ${ }^{18}$

21 (a) Iverson, D. J.; Hunter, G.; Blount, J. F.; Damewood, Jr., J. R.; Mislow, K. J. Am. Chem. Soc. 1981, 103, 6073. (b) See, also: Hunter, G.; Iverson, D. J; Mislow, K.; Blount, J. F. J. Am. Chem. Soc. 1980, 102, 5942. (c) Hunter, G.; Weakley, J. R.; Mislow, K.; Wong, M. G. J. Chem. Soc., Dalton Trans. 1986, 577.

22 The sharply decreasing trend in the values of $K_{\mathrm{DAECT}}$ relates to the corresponding changes in color intensities arising from the chloranil complexes with MES, TXY, and DTT in Table 3. Such differences in color intensity result mainly from the variations of $\varepsilon_{\mathrm{C} T}$ since the values of $K_{\mathrm{DA}}$ are relatively invariant.

23For the relationship that connects the separation $(d)$ with $\lambda_{c t}$ and $\varepsilon_{C T}$, see: footnote 5 .

$\underline{\mathbf{2 4}}$ (a) The barrier to the rotation of the aryl-ethyl bond in HEB has been estimated to be 11.8 $\mathrm{kcal} \mathrm{mol}^{-1} .^{21 \mathrm{a}}$ (b) The reduced overlap of the donor-acceptor $\pi$-orbitals as a result of such a parallel shift is reflected in a corresponding decrease in the $\varepsilon$ cт values with increasing number of tert-butyl groups in Table 3 (see entries 1-4). (c) According to Mulliken, ${ }^{4 a}$ the charge-transfer intensity (KDAECT) derives from the transition moment which relates to the overlap integral of the donor and acceptor orbitals (see: Orgel, L. E.; Mulliken, R. S. J. Am. Chem. Soc. 1957, 79, 4839.

25 See: Staab, M. A.; Reibel, W. R. K.; Krieger, C. Chem. Ber. 1985, 118, 1230.

$\underline{26}$ The results in Table 3 (last 3 entries) show a large decrease in the values of $K_{\mathrm{DAECT}}$ despite the opposite trend in the donor properties $\left(E_{0 x}{ }^{\circ}\right)$ of ME, MEA, and MEA2. Such a decrease in the effective absorbance, without large changes in $K_{\mathrm{DA}}$, is attributed to the reduced overlap of the donor and acceptor $\pi$-orbitals ${ }^{24 c}$ due to the steric shielding of the dimethoxy-substituted benzene ring by the norbornane framework.

27 See: Foster, R. in ref 4 for the relationship between the donor strength and $E_{0 x^{\circ}}{ }^{\circ}$ as measured electrochemically (see Experimental Section).

$\underline{28}$ Molecular modeling calculations predict an optimum separation of $\sim 4 \AA$.

29 Maverick, E.; Trueblood, K. N.; Bekoe, D. A. Acta Crystallogr., Sect. B 1978, 34, 2777.

30 Kim, E. K.; Kochi, J. K. J. Am. Chem. Soc. 1991, 113, 4962.

31 X-ray crystallographic analysis of various arene/ $/ \mathrm{NO}^{+}$complexes, ${ }^{32}$ generally showed the nitrosonium to be sufficiently disordered to obscure the distinction between $\mathrm{N}$ and $\mathrm{O}$. On the other hand, HEB and TET complexes showed no such disorder, and $\mathrm{N}$ bonding to the aromatic donor could be readily established.

32 (a) Brownstein, S.; Gabe, E.; Lee, F.; Tan, L. J. Chem. Soc., Chem. Commun. 1984, 1566. (b) Brownstein, S.; Gabe, E.; Lee, F.; Piotrowski, A. Can. J. Chem. 1986, 64, 1661. (c) Brownstein, S.; Gabe, E.; Irish, B.; Lee, F.; Louie, B.; Piotrowski, A. Can. J. Chem. 1987, 65, 445. (d) Borodkin, G. I.; Nagi, S. M. Gatilov, Y. V.; Mamatyuk, V. I.; Mudrakovskii, T. L.; Shubin, V. G. Dokl. Akad. Nauk SSSR 1986, 288, 1364. (e) Kim, E. K.: Kochi, J. K. J. Org. Chem. 1993, 58, 786. 
33 Despite the steric encumbrance in the HEB and TET complexes, the magnitude of the separation of $d=2.08 \AA$ is not significantly different from that found in the HMB complex with $\mathrm{NO}^{+}$.

34 The van der Waals diameters of the cavities in HEB and TET were estimated to be $\sim 3.8$ and 3.6 $\AA$, respectively, based on the published structures. ${ }^{9,21 a}$

35 Sankararaman, S.; Haney, W. A.; Kochi, J. K. J. Am. Chem. Soc. 1984, 109, 5235 and 7824. Also, see: Kochi, J. K. Acta Chem. Scand. 1990, 44, 409.

36 Although the "center" of $\mathrm{NO}_{2}$ as the acceptor moiety in TNM is unclear, an estimate of the separation is given by calculated distances of 2.9 and $3.0 \AA$ of the oxygen pair to the aromatic plane in $\mathbf{T}$. The corresponding distances in $\mathbf{U}$ are 3.3 at $3.6 \AA$.

37 Pasimeni, L.; Guella, G.; Corvaja, C.; Clemente, D. A; Vicentini, M. Mol. Cryst. Liq. Cryst. 1983, 91, 25.

38 Note that the presence of a pair of ortho substituents in PM-T and PE-T essentially precludes a planar biphenyl framework as in structure $\mathbf{V}$.

39 (a) The van der Waals radii of chloranil and benzene are 1.8 and $1.7 \AA$, respectively (see: Pauling, L. The Nature of the Chemical Bond, 3rd ed.; Cornell: Ithaca, NY 1960; $p$ $257 \mathrm{ff})$. We approximate the van der Waals radius of the hexamethylbenzene moiety in structure A to be slightly less $(\sim 1.8 \AA)$ than that of the methyl groups owing to the staggering of the $\mathrm{Cl}$ and $\mathrm{CH}_{3}$ groups as well as the preferred conformation of the methyl groups (as a result of restricted rotation), as shown in structure $\mathbf{A}_{\text {T. }}$ (b) The inner-sphere character is cleanly delineated in the nitrosonium/arene complexes $\mathbf{Q}, \mathbf{R}$, and $\mathbf{S}$ by the (nonbonded) nitrogen-arene distance of $\sim 2.1 \AA$, which is substantially less than the sum of the van der Waals radii of benzene and nitrosonium (3.2 $\AA$ ).

40 Rathore, R.; Bosch, E.; Kochi, J. K. J. Chem. Soc., Perkin Trans 2 1994, 1157.

41 Rathore, R.; Kochi, J. K. J. Org. Chem. 1995, 60, 4399.

42 Halterman, R. L.; Jan. S.-T. J. Org. Chem. 1991, 56, 5253.

43 Bruson, H. A.; Kroeger, J. W. J. Am. Chem. Soc. 1940, 62, 36.

44 Kong, K.-C.; Cheng, C.-H. J. Chem. Soc., Chem. Commun. 1991, 423.

45 Tamao, K.; Sumitani, K.; Kiso, Y.; Zembayashi, M.; Fujioka, A.; Kodana, S.; Nakayima, I.; Minato, A.; Kumada, M. Bull. Chem. Soc. Jpn. 1976, 49, 1958.

46 See: Komatsu et al. in ref 9.

47 Gassman, P. G.; Gennick, I. J. Am. Chem. Soc. 1980, 102, 6863.

$\underline{48}$ Downton, P. A.; Milvaganam, B.; Frampton, C. S.; Sayer, B. G.; McGlinchey, M. J. J. Am. Chem. Soc. 1990, 112, 27.

49 van der Made, A. W.; van der Made, R. I. J. Org. Chem. 1993, 58, 1262.

50 Takahashi, Y.; Sankararaman, S.; Kochi, J. K. J. Am. Chem. Soc. 1989, 111, 2954.

51 See: Person, W. B. in ref $16 \mathrm{~b}$.

$\underline{52}$ Gasteiger, J.; Marsili, M. Tetrahedron 1980, 36, 3219.

$\underline{53}$ Press, W. H.; Flannery, B. P.; Teukolsky, S. A.; Vetterling, W. T. Numerical Recipes in C. The Art of Scientific Computing; Cambridge University Press: New York, 1988; p 317.

54 Brooks, B. R.; Bruccoleri, R. E.; Olafson, B. D.; States, D. J.; Swaminathan, S.; Karplus, M. J. Comput. Chem. 1983, 4, 187.

55 Sheldrick, G. M. SHELSX-86, Program for Structure Solution; University of Göttingen: Germany, 1986.

$\underline{56}$ XP-Interactive Molecular Graphics (vers. 5.06); Siemens Analytical Instruments: Madison, WI, 1996. 